\title{
Contribuzione alla teoria delle 27 rette e dei 45 piani tritangenti di una superficie di $3^{\circ}$ ordine.
}

(Memoria del prof. E. Bertini, a Pavia.)

Il presente lavoro è diviso nei paragrafi seguenti $\left(^{*}\right)$ :

$\S$ 1. Relazioni fra le coppie di triedri conjugati. $-\S 2$. Enneaedri di $1^{a}$ e 2 ' specie. - $\$ 3$. Relazioni fra le terne T. $-\S 4$. Ordinamento e costruzione delle terne $T$. $-\S 5$. Teoremi sugli enneaedri. $-\S 6$. Triedri di $1^{2}$, $2^{\mathrm{a}}$ e $3^{\mathrm{a}}$ specie. Altre proprietà degli enneaedri. $-\S 7$. Poliedri. Pentaedro principale. $-\S 8$. Ettaedro principale. $-\S 9$. Poliedri che contengono almeno un triedro di $1^{a}$ specie. $-\S 10$. Tetraedri. $-\$ 11$. Pentaedri. $-\$ 12$. Esaedri. - § 13. Ettaedri. Ottaedri. - $\$ 14$. Specchio riassuntivo dei poliedri.

Per brevità si chiama terna $T$ il sistema di tre coppie di triedri conjugati, nelle quali giacciono tutte le 27 rette della superficie. Di queste terne $T$ e delle coppie di triedri conjugati, i $\S \S 1,3,4$ contengono alcune nuove proposizioni, le quali in seguito somo di ajuto in parecchi punti. Nel $\S 4$ è esposta inoltre la costruzione delle 40 terne $T$. Benchè tale costruzione non abbia interesse per la parte rimanente del lavoro, tuttavia è parso opportuno di non ommetterla per mostrare come le proprietà premesse e la arbitrarietà inerente alla notazione fossero sufficienti per quella costruzione. I $\S \S 2,5$ sono consacrati a dimostrare varie proprietà degli enneaedri. Alcune di queste proprietà $e$ in particolare la distinzione degli enneaedri in due specie, come si avverte a suo luogo, sono dovute a Cremora. Le dimostrazioni date in quei due para-

(') La maggior parte dei nuovi risultati contenuti nei $\$ \S 1,2, \ldots 9$, furono comunicati, senza dimostrazione, al R. Istituto Lombardo, nelle sedute del 15 maggio e 31 luglio 1884. 
grafi non sono fondate sopra alcuna notazione delle 27 rette o dei 45 piani tritangenti (nemmeno su quella del n. ${ }^{\circ} 11$ ).

Nel $\S 6$ si pone il concetto generale di triedro e si distinguono tre sorta di triedri: alle quali cose si collegano alcune nuove proprietà degli enneaedri.

I paragrafi seguenti $7,8, \ldots 14$ sono rivolti alla risoluzione di un problema, che sino ad oggi non fu oggetto di indagine. Quali e quanti sono i poliedri (di cui gli spigoli sono esterni alla superficie) che si possono formare coi 45 piani tritangenti? Si arriva alla risoluzione di questo problema col determinare dapprima i poliedri principali, ossia quelli non contenuti in altri poliedri. Di poliedri principali esistono quattro specie; cioè, oltre le due specie di enneaedri, una specie di ettaedri $(\S 8)$ e una di pentaedri $(\S 7)$ : quella non considerata, credo, finora; questa incontrata da CREMona per altro scopo (cfr. note al $n .^{\circ} 23$ ). Nel $\S 8$ che si riferisce agli ettaedri principali, e in quello soltanto, si ricorre, cercando però di mantenere la generalità della ricerca, alla notazione di Schlaefl, per evitare una discussione che si presenta molto complicata. In appresso, noti i poliedri principali, si ricercano i poliedri in essi contenuti e così si trovano tutti i possibili tetraedri, pentaedri...; i quali sono poi raccolti in uno specchio con alcuni numeri relativi $(\$ 14)$. In questa seconda parte della ricerca s'incontra la difficoltà di riconoscere se poliedri contenuti in differenti poliedri principali e aventi certi caratteri comuni, sieno o no diversi. La risoluzione di tale difficoltà porta a concludere che un poliedro di una data specie c̀ definito da quattro numeri: il numero dei piani che lo formano ed i tre numeri che indicano quanti triedri delle tre specie si possono formare coi piani stessi.

\section{§ 1. Relazioni fra le coppie di triedri conjugati.}

1. Indichi

$$
C \equiv\left|\begin{array}{lll}
a_{11} & a_{13} & a_{13} \\
a_{21} & a_{22} & a_{23} \\
a_{31} & a_{32} & a_{33}
\end{array}\right| \equiv\left|\begin{array}{ll}
a_{1} & b_{1} \\
a_{2} & b_{2} \\
a_{3} & b_{3}
\end{array}\right|
$$

una coppia qualsiasi di triedri conjugati, essendo $a_{11}, a_{12}, a_{13}, a_{21}, \ldots$ le nove rette di essa e

$$
\begin{aligned}
& a_{i} \equiv a_{i 1} a_{i 2} a_{i 3} \\
& b_{i} \equiv a_{1 i} a_{2 i} a_{3 i}
\end{aligned} \quad i=1,2,3
$$


i sei piani della medesima (*). Cerchiamo le coppie che hanno colla $C$ elementi (piani o rette) comuni.

Si sa e si dimostra facilmente che una terna gobba $\left(^{* *}\right)$ di rette individua una coppia di triedri conjugati, di cui le sei rette rimanenti sono le bisecanti della terna.

È noto altresi $(* * *)$ che due piani, aventi una retta comune, appartengono a quattro coppie di triedri conjugati. Ora coi sei piani della coppia $C$ si possono formare nove delle dette paja di piani. Si hanno adunque 27 coppie di triedri conjugati che hanno colla $C$ due piani comuni.

Si consideri adesso una coppia gobba di rette di $C$. $\grave{\mathrm{E}}$ chiaro che essa compare in sei delle precedenti 27 coppie di triedri conjugati (per es., $a_{21}, a_{12}$ entrano nelle tre coppie contenenti i piani $a_{1}, b_{1}$ e nelle tre contenenti i piani $\left.a_{2}, b_{2}\right)$. Ma una coppia gobha di rette fa parte di dieci terne gobbe di rette. Escludendo le sette terne gobbe, che individuano $C$ e le sei coppie di triedri conjugati testè nominate, rimangono tre terne che dànno tre coppie di triedri conjugati aventi a comune con $C$ la considerata coppia gobba di rette (e, come è facile vedere, null'altro). Cosicchè, in totale, si hanno $18 \cdot 3=54$ coppie di triedri conjugati che hanno comune con $C$ una coppia gobba di rette (soltanto).

I piani che non contengono alcuna delle nove rette della coppia $C$ sono manifestamente

$$
45-3 \cdot 9-6=12 .
$$

Uno $a_{1}$ di questi dodici piani e un piano $\alpha_{1}$ di $C$ dànno origine ad una coppia $C^{\prime}$ di triedri conjugati. Il terzo piano che con $\alpha_{1}, a_{1}$ forma un triedro di $C^{\prime}$ deve essere degli stessi dodici piani; giacchè, altrimenti, conterrebbe almeno una retta di $C$, la quale, insieme ad una retta di $a_{1}$, darebbe un nuovo piano (oltre $a_{1}$ ) comune a $C, C^{\prime}$, onde $\alpha_{1}$ dovrebbe contenere una retta di $C$. Quei dodici piani determinano adunque sei coppie di triedri conjugati che hanno con $C$ a comune il solo piano $a_{1}$. E si hanno quindi 36 coppie di triedri conjugati aventi con $C$ un solo piano comune.

2. E qui si noti che, una retta appartenendo a 16 coppie gobbe e una coppia gobba a 10 terne gobbe, una retta entra in 80 terne gobbe e però in

() Qui e dappertutto in seguito, piani e rette sono (se nulla si avverta in contrario) piani tritangenti e rette di una superficie generale del $3^{\circ}$ ordine.

(“) L'irsieme di più rette dicesi gobbo se due qualunque di esse non s'incontrano.

(**) R. STuRm, Synthetische Untersuchungen über Flächen dritter Ordnung, Leipzig, 1867, pag. 64 . 
40 coppie di triedri conjugati (*). Le 39 coppie di triedri conjugati, che hanno con $C$ una retta comune, si distribuiscono in tre gruppi di 12, 12, 15, aventi rispettivamente a comune con $C$ una coppia gobba di rette, un piano, due piani. Il che è evidente per le cose dette nel n. ${ }^{\circ} 1$ e arvertendo che una retta di $C$ appartiene a quattro coppie gobbe, a due piani, e a cinque coppie di piani (aventi una retta comune) contenuti in $C$.

Quindi non possono due coppie di triedri conjugati avere una sola retta comune.

Ne discende facilmente la proprietà nota che, esistono due coppie di triedri conjugati (e due soltanto) che, con una data coppia $C$, contengono tutte le 27 rette (**). In vero, escludendo le nove rette di $C$ e i piani che passano per esse, rimangono 12 piani e 18 rette. Ciascuna di queste rette esiste in due dei 12 piani, giacchè incontra $i$ tre piani di un triedro di $C$ sopra tre rette di $C$, formanti una terna gobba, colle quali dà tre piani esclusi. Sia $\alpha_{1}$ uno qualunque dei 12 piani e $\beta_{1}, \beta_{2}$ quelli di essi che passano per due rette di $\alpha_{1}$ (oltre $\alpha_{1}$ ). I piani $\beta_{1}, \beta_{2}$ individuano una coppia $C^{\prime}$, di cui un triedro sarà formato dagli stessi piani $\beta_{1}, \beta_{2}$ e da un terzo piano che diremo $\beta_{3}$ e l'altro triedro conterrà $\alpha_{1}$. Nella coppia $C^{\prime}$ entrano, delle 18 rette suddette, tre di $\beta_{1}$, tre di $\beta_{2}$ e la retta $\alpha_{1} \beta_{3}$. Segue, per l'ultima proprietà avvertita, che le coppie $C, C^{\prime}$ non hanno alcuna retta o piano comune: cioè $C^{\prime}$ contiene sei dei 12 piani e nove delle 18 rette. Prendasi ora un altro dei 12 piani, all'infuori dei sei di $C^{\prime}$, e si avrà una nuova coppia che conterrà i sei piani e le nove rette rimanenti, poichè per una retta di $C^{\prime}$ passano due soli dei 12 piani e sono quelli della stessa coppia $C^{\prime}$. Adunque, ecc.

3. Riunendo l'ultima proprietà a quelle del $\mathrm{n}^{\circ} 1$, si ha il seguente teorema:

Rispetto ad una coppia di triedri conjugati, le altre 119 coppie si distribuiscono in quattro gruppi:

$1 .^{\circ}$ Di 2 coppie, che nulla hanno a comune colla data (e fra loro);

$2 .^{\circ}$ Di 27 coppie, ciascuna delle quali ha colla data due piani comuni (e cinque rette);

$3 .^{\circ} \mathrm{Di} 36$ coppie, ciascuna delle quali ha colla data un piano comune (e tre rette);

() Cid segue subito anche dall' osservare che 27.40=120.9. Veggasi inoltre Sturm, l. c.

(“) J $\mathrm{J}_{\mathrm{ACOB}}$ Steiner's, Gesammelte Werke, Berlin, 1882, tom. II, pag. 655. - Sturm, 1. c., pag. 63 . - Cremona-Curtze, Oberfü̈chen, $\$ 261$. 
4. Di 54 coppie, cadauna avente comune colla data una coppia gobba di rette (e nessun piano).

Il sistema di tre coppie di triedri conjugati che contengono insieme le 27 rette della superficie, si chiamerà d'ora innanzi una terna $T$.

4. Sia

\section{$\S 2$. Enneaedri di $1^{a}$ e $2^{a}$ specie.}

$$
T^{\prime} \equiv\left|\begin{array}{ll|ll|ll}
a_{1} & b_{1} & a_{1} & b_{4} & a_{7} & b_{7} \\
a_{2} & b_{2} & a_{5} & b_{5} & a_{8} & b_{8} \\
a_{3} & b_{3} & a_{8} & b_{6} & a_{9} & b_{9}
\end{array}\right|
$$

una terna di coppie di triedri conjugati, ove $a_{1} a_{2} a_{3}$ indica un triedro, $b_{1} b_{2} b_{3}$ il triedro ad esso conjugato, ecc. Ciascuno dei 27 piani esclusi dalla terna $T^{\prime}$ contiene una retta di ogni coppia di $T^{\prime}$, perchè due rette di una stessa coppia, che s'incontrano, dànno un piano della coppia. Presi ad arbitrio due piani (ad es.) $a_{1}, a_{4}$ di due coppie di $T^{\prime}, \mathrm{i}$ tre piani che le rette dell' uno formano colle rette dell'altro, sono dei suddetti 27 piani e però contengono tre nuove rette appartenenti alla terza coppia di $T^{\prime}$. Cioè il triedro, a cui i piani $a_{1}, a_{4}$ dànno origine, è completato da un piano della terza coppia di $T^{\prime}$, per es. (potendosi, nell'indicazione di $T^{\prime}$, scambiare due colonne di una matrice) da uno dei piani $a_{7}, a_{8}, a_{9} . \mathrm{E}$, ciò essendo, il triedro individuato dai piani $a_{1}, a_{5}$ deve essere completato da uno degli stessi tre piani $a_{7}, a_{8}, a_{9}$; perchè, esistendo, ad es., i triedri $a_{1} a_{4} a_{7}, a_{1} a_{5} b_{7}$, si avrebbero due coppie di triedri conjugati con quattro (sole) rette comuni, cioè le tre rette di $a_{1}$ e la retta $a_{7} b_{7}$, il che non può essere (n..$^{\circ}$ ). La stessa considerazione prova che i triedri originati dalle coppie di piani $a_{1}, a_{6} ; a_{2}, a_{4} ; a_{2}, a_{5} ; a_{2}, a_{6} ; a_{3}, a_{4} ; a_{3}, a_{5} ; a_{3}, a_{6}$ sono completati da uno degli stessi tre piani $a_{7}, a_{8}, a_{9}$. Se ne ricava immediatamente che il sistema dei nove piani

$$
a_{1} a_{2} a_{3} a_{4} \ldots a_{9}
$$

gode della proprietà che, assunti ad arbitrio due dei nove piani, il terzo piano che con essi completa il triedro $\dot{e}$ degli stessi nove piani. Per conseguenza, quei nove piani si possono distribuire in 12 triedri, ogni piano appartenendo a 4 triedri: e inoltre, $i 12$ triedri si spartiscono in 4 terne, ogni terna contenendo tutti $i$ nove piani $\left(E_{1}\right)\left({ }^{*}\right)$.

(") Cremona, Sulle 27 rette di una superficie di $3^{\circ}$ ordine, Rendiconti del R. Istituto Lombardo, Serie II, rol. $3^{\circ}$, pag. 216. 
Per giungere facilmente ad una opportuna notazione di quei 12 triedri, si può avvertire altresì questa proprietà. Se un piano $a_{i}$ è comune a due triedri (dei 12 suddetti),_ $i$ quattro piani residui di questi triedri non possono appartenere a due nuovi triedri che abbiano pure un piano comune $a_{j}$. Perocchè, se ciò avvenisse, aggiungendo ai triedri qui considerati il triedro dato dai piani $a_{i}, a_{j}$, tre dei triedri, a cui appartiene $a_{i}$ sarebbero formati degli stessi sette piani di tre dei triedri a cui appartiene $a_{j}$; e quindi i due piani rimanenti dovrebbero formare un triedro tanto con $a_{i}$ che con $a_{j}$, ciò che non può essere.

Potendosi nell' indicazione di $T^{\prime}$ scambiare due piani di una colonna, si può fissare che $\mathrm{i}$ triedri, a cui appartiene $a_{1}$, sieno (oltre $\left.a_{1} a_{2} a_{3}\right) a_{1} a_{4} a_{7}, a_{1} a_{5} a_{9}$, $a_{1} a_{6} a_{8}$. Allora il triedro, di cui fanno parte $a_{2}, a_{1}$, non può essere completato che da $a_{8}$ o da $a_{3}$ e può ritenersi sia il triedro $a_{2} a_{1} a_{3}$ (scambiando, quando non fosse, $a_{8}$ con $a_{9}, a_{6}$ con $a_{5}$ ). Ciò fatto, la notazione di tutti gli altri triedri è determinata. In vero il triedro dato da $a_{2}, a_{5}$ non può essere completato da $a_{9}$ manifestamente, e nemmeno da $a_{7}$, giacchè, in questo caso, i due triedri $a_{2} a_{5} a_{7}, a_{2} a_{4} a_{9}$ arrebbero un piano comune e $\mathrm{i}$ quattro piani residui darebbero i due nuovi triedri $a_{1} a_{4} a_{7}, a_{1} a_{5} a_{9}$ pure con un piano comune. Si ha quindi il triedro $a_{2} a_{5} a_{8}$ : e allora si ottiene necessariamente il triedro $a_{2} a_{6} a_{8}$, ecc. In tal modo i 12 triedri risultano dati e distribuiti nelle quattro terne, per le orizzontali, le verticali, $\mathrm{i}$ termini positivi e i negativi del determinante

$$
\left|\begin{array}{lll}
a_{1} & a_{2} & a_{3} \\
a_{4} & a_{5} & a_{6} \\
a_{7} & a_{8} & a_{9}
\end{array}\right| .
$$

5. Si osservi ora, continuando a considerare i nove piani $\left(E_{1}\right)$, che essi appartengono a quattro terne $T$ (fra le quali $T^{\prime}$ ). Infatti segue immediatamente dalla proprietà del $\mathrm{n}^{\circ} 3$, che tre triedri che non hanno rette comuni (ciò̀ contengono tutte le 27 rette) sono di una terna $T$ e rispettivamente delle sue tre coppie, giacchè, per quella proprietà, due triedri, non appartenenti alla stessa terna $T$, hanno rette comuni. Può aggiungersi che le quattro terne $T$, a cui appartiene il sistema $\left(E_{1}\right)$, contengono tutti i 45 piani tritangenti; cioè i 36 muovi piani, oltre $i$ piani del sistema, che entrano in esse, sono tutti

(") Le proprieta precedenti sono identiche a ruelle dei flessi di una curva di $3^{\circ}$ ordine, so si sostituisca rispettivamente a piano e triedro, flesso e retta dei flessi: cioè i nove piani $\left(E_{1}\right)$ sono dati da una equazione hessiana. 
distinti. Giacchè due coppie qualunque appartenenti rispettivamente a due di quelle quattro terne, per es. le coppie date dai triedri $a_{1} a_{2} a_{3}, a_{1} a_{4} a_{7}$, hanno a comune un piano $a_{1}$ e uno solo, le rette di $a_{2}, a_{3}$ essendo distinte da quelle di $a_{4}, a_{7}(*)$.

Se si considera invece il sistema dei nove piani che si ottengono da $\left(E_{1}\right)$ col sostituire ad un triedro il suo conjugato, ad es., il sistema

$$
b_{1} b_{2} b_{3} a_{4} a_{5} a_{6} a_{7} a_{8} a_{9}
$$

(formato dalla $2^{\mathrm{a}}, 3^{\mathrm{a}}, 5^{\mathrm{a}}$ colonna di $T^{\prime}$ ), si hanno ancora nove piani contenenti tutte le 27 rette della superficie, ma qui non esistono altri triedri, all' infuori dei tre, $b_{1} b_{2} b_{3}, a_{4} a_{5} a_{6}, a_{7} a_{5} a_{9}$. Infatti uno dei piani $a_{4}, a_{5}, a_{6}$ e uno dei piani $a_{7}, a_{8}, a_{0}$ dànno un triedro, che è completato da uno dei piani $a_{1}$,

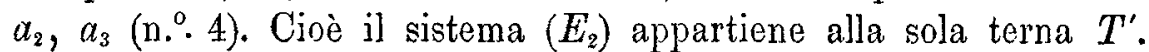

Adunque, se dicasi enneaedro il sistema di nove piani tritangenti nei quali giacciono tutte le 27 rette della superficie, si hanno due specie di enneaedri: quelli di $1^{a}$ specie, che si spezzano in 4 modi in tre triedri, ossia appartengono a quattro terne $T$ e quelli $d i 2^{a}$ specie che si spezzano in un modo solo in tre triedri, cioè appartengono ad una sola terna $T$.

(") Se si indica con $a_{l}^{(i)} a_{m}^{(i)} \alpha_{n i}^{(i)}$ il triedro conjugato ad $a_{l} a_{m} a_{n}$, ponendo ordinatamente $i=1, i=2, i=3, i=4$ per i triedri conjugati ai triedri dati dalle orizzontali, dalle verticali, dai termini positivi e dai negativi del determinante del n. ${ }^{\circ} 4$ e inoltre si rappresentano colle stesse lettere i primi membri delle eçuazioni dei piani dei triedri, moltiplicate per. opportune costanti, l'equazione della superficie di $3^{\circ}$ ordine si può scrivere nelle forme seguenti:

$$
\begin{aligned}
& a_{1} a_{2} a_{3}=a_{1}^{(1)} a_{2}^{(2)} a_{3}^{(3)}, \\
& a_{4} a_{5} a_{6}=a_{4}^{(1)} a_{5}^{(1)} a_{5}^{(1)}, \\
& a_{7} a_{8} a_{9}=a_{7}^{(1)} a_{8}^{(1)} a_{9}^{(1)}, \\
& a_{1} a_{4} a_{7}=a_{1}^{(2)} a_{4}^{(2)} a_{7}^{(2)}, \\
& \ldots \ldots \ldots \ldots \ldots \\
& a_{1} a_{6} a_{8}=a_{1}^{(4)} a_{6}^{(4)} a_{8}^{(4)}, \\
& a_{2} a_{4} a_{3}=a_{2}^{(4)} a_{4}^{(4)} a_{9}^{(1)} ;
\end{aligned}
$$

donde l'equazione data dal Cremona (l. c., nei Rendiconti del R. Ist. Lomb., pag. 216)

$$
\left(a_{1} a_{2} a_{3} \ldots a_{9}\right)^{5}=\Pi,
$$

essendo $\Pi$ il prodotto dei 45 piani tritangenti; ecc. 
Una terna $T$ contiene quattro enneaedri di $1^{\text {a }}$ specie e quattro di $2^{2}$. Per es., la terna $T^{\prime}$, oltre $\left(E_{1}\right)$, contiene gli enneaedri di $1^{2}$ specie

$$
\begin{aligned}
& a_{1} a_{2} a_{3} b_{4} b_{5} b_{6} b_{7} b_{8} b_{9}, \\
& b_{1} b_{2} b_{3} a_{4} a_{5} a_{6} b_{7} b_{8} b_{9}, \\
& b_{1} b_{2} b_{3} b_{4} b_{5} b_{6} a_{7} a_{8} a_{9}
\end{aligned}
$$

poichè il triedro dato da $a_{1}, b_{4}$ (ad es.) deve essere completato da uno dei piani $b_{7}, b_{8}, b_{9}$, non potendo esserlo da uno dei piani $a_{7}, a_{8}, a_{9}$ (cfr. n. ${ }^{\circ} 4$ ), ecc. E la stessa terna $T^{\prime}$ contiene, oltre $\left(E_{2}\right)$, gli enneaedri di $2^{\mathfrak{z}}$ specie

$$
\begin{aligned}
& a_{1} a_{2} a_{3} b_{4} b_{5} b_{6} a_{7} a_{8} a_{9}, \\
& a_{1} a_{2} a_{3} a_{4} a_{5} a_{6} b_{7} b_{8} b_{7}, \\
& b_{1} b_{2} b_{3} b_{4} b_{5} b_{6} b_{7} b_{8} b_{9},
\end{aligned}
$$

che provengono dai precedenti sostituendo ad uno o a tre triedri i loro conjugati.

Per conseguenza nascono, dalle 40 terne $T, \frac{40 \cdot 4}{4}=40$ enneaedri di $1^{3}$ specie e $40 \cdot 4=160$ enneaedri di $2^{2}$ specie $\left(^{*}\right)$.

6. Questi enneadri sono $i$ soli che si possono formare coi 45 piani tritangenti. In vero sia

$$
x_{1} x_{2} \ldots x_{9}
$$

un enneaedro, cioè il sistema di nove piani che contengono le 27 rette e che non hanno quindi rette (della superficie) a comune. Un piano qualunque, $x_{1}$ (ad es.), deve appartenere almeno ad un triedro formato di piani dell'enneaedro. Poichè, se ciò non fosse, combinando $x_{1}$ cogli altri otto piani dell'enneaedro, si avrebbero otto triedri e quindi otto coppie con un piano comune $x_{1}$, e con questo solo, per la considerazione che, quando due coppie di triedri hanno due piani comuni, ogni altro piano di una coppia ha una retta comune con ogni altro piano dell'altra coppia. Il che non può essere, rispetto ad una coppia esistendo (n. ${ }^{\circ}$ ) sei sole altre coppie che hanno colla medesima un (solo) dato piano comune. Ora, se i piani $x_{1}, x_{2}, x_{3}$ (ad es.) formano un triedro, i piani che non contengono rette di essi sono 12 e si distribuiscono in due coppie

(') Cremona, 1, c. (nei Rendiconti del R. Istituto Lombardo), rag. 215. 
appartenenti alla terna $T$ individuata del triedro $x_{1} x_{2} x_{3}\left(\mathrm{n}^{\circ}{ }^{0} 2\right)$. Cioè gli altri sei piani $x_{4} x_{5} \ldots x_{9}$ sono di quella terna: c. d. d. $\left({ }^{*}\right)$.

7. Dall'ultima considerazione del $n .^{0} 6$ segue che due enneaedri (di $1^{2} o$ $2^{2}$ specie) aventi un triedro comune appartengono alla medesima terna $T$, quella individuata dal triedro: e viceversa, per le cose del $n^{\circ} 5$, che un triedro è comune a due enneaedri (della stessa specie) e a due soli appartenenti ad una medesima terna.

Dal $\mathrm{n} .{ }^{\circ} 5$ si ha inoltre manifestamente che $i$ quattro enneaedri di una stessa specie, nascenti da una terna $T$, sono tali che due qualunque hanno un triedro comune e $i$ due rimanenti hanno comune il triedro conjugato: ossia, $i$ quattro enneaedri si possono decomporre ciascuno in tre triedri, per modo che $i$ triedri dell' uno appartengano rispettivamente. agli altri tre. Questa proprietà vale non soltanto per gli enneaedri di $1^{\mathrm{a}}$ specie, come fu osservato dal Cremona $\left({ }^{* *}\right)$, ma anche per quelli di $2^{\mathrm{a}}$ specie. La differenza sta in cio, che ogni enneaedro di $1^{2}$ specie appartiene a quattro di quelle quaderne, mentre ognuno di $2^{2}$ ad una sola. Onde si hanno 40 delle dette quaderne (tante, quante le terne $T$ ) si degli enneaedri di $1^{\alpha}$ specie, come degli enneaedri di $2^{a}$.

\section{§3. Relazioni fra le terne $T$.}

8. Da una terna $T^{\prime}$ (di cui si conserva la notazione data nel n. ${ }^{\circ}$ 4) nascono quattro enneaedri di $1^{\text {a }}$ specie, ognuno dei quali esiste in tre nuove terne; cosicchè, rispetto ad una terna $T^{\prime}$, esistono 12 altre terne $T$, che hanno con

() Introducendo la notazione di Schlakfli, gli enneaedri sono dei due tipi:

$\begin{array}{lllllll}a_{i} & b_{k} & c_{i k} & & a_{i} & b_{k} & c_{i k} \\ a_{k} & b_{l} & c_{k l} & & a_{k} & b_{l} & c_{k l} \\ a_{l} & b_{i} & c_{l i} & a_{l} & b_{m} & c_{l n} \\ a_{m} & b_{n} & c_{m n} & a_{m} & b_{n} & c_{m n} \\ a_{n} & b_{p} & c_{n p} & a_{n} & b_{p} & c_{m p} \\ a_{p} & b_{m} & c_{p m} & & a_{p} & b_{i} & c_{p i} \\ c_{i m} & c_{k n} & c_{l p} & & c_{i l} & c_{m p} & c_{k n} \\ c_{m n} & c_{k p} & c_{l m} & & c_{2 n} & c_{p k} & c_{i m} \\ c_{i p} & c_{k m} & c_{l n} & & c_{m i} & c_{k m} & c_{l p}\end{array}$

essendo $i k l m n p$ una permutazione qualunque dei numeri $12 \ldots 6$. Se ne hanno 80 del $1^{\circ}$ tipo (fra $\mathrm{i}$ quali $\mathrm{i} 40$ enneaedri di $1^{\mathrm{a}}$ specie) e 120 del $2^{\circ}$ tipo.

(") Cremona, 1. c. (nei Rendiconti del R. Istituto Lomkardo), pag. 219. 
quella un enneaedro di $1^{\mathrm{a}}$ specie a comune. Cerchiamo in quale relazione stanno alla terna $T^{\prime}$ le rimanenti 27 terne; di cui una qualsiasi si dirà $T^{\prime}{ }_{1}$. Si consideri una coppia di $T^{\prime}$, per es., la

$$
\left|\begin{array}{ll}
a_{1} & b_{1} \\
a_{2} & b_{2} \\
a_{3} & b_{3}
\end{array}\right| .
$$

Un suo piano $a_{1}$ qualsivoglia, appartenendo a due enneaedri di $1^{\text {a }}$ specie nascenti da $T^{\prime}$ (cioè ad $a_{1} a_{2} \ldots a_{9}, a_{1} a_{2} a_{3} b_{4} \ldots b_{9}$ ), entra in 6 delle 12 terne $T$ escluse, cioè in sei coppie appartenenti a tali terne: e queste sei coppie non hanno con $\left(C^{\prime}\right)$ altro piano comune, oltre $a_{1}$ (cfr. n. ${ }^{\circ} 5$ ). Adunque le 36 coppie (n. ${ }^{\circ}$ ) che hanno con $\left(C^{\prime}\right)$ un (solo) piano comune sono delle 12 terne $T$ escluse. Ossia una coppia di $T^{\prime}$ e una di $T^{\prime}{ }_{1}$ avranno a comune due piani 0 nessuno. Per fissare le idee, sia

$$
\left|\begin{array}{ll}
c_{1} & d_{1} \\
c_{2} & d_{2} \\
c_{3} & d_{3}
\end{array}\right|
$$

una coppia di $T^{\prime}{ }_{1}$. Se essa ha con $\left(C^{\prime}\right)$ due piani comuni $a_{1} \equiv c_{1}, b_{1} \equiv d_{1}$, nessun piano deve avere a comune colle altre due coppie di $T^{\prime}$, per es., colla

$$
\left|\begin{array}{ll}
a_{5} & b_{5} \\
a_{6} & b_{6} \\
a_{7} & b_{7}
\end{array}\right| ;
$$

giacchè, se fosse $a_{5} \equiv c_{2}, b_{5} \equiv d_{2}$, le due coppie $(C)\left(C^{\prime \prime \prime}\right)$, della stessa terna $T^{\prime}$, arrebbero a comune le rette $c_{1} d_{2} \equiv a_{1} b_{5}, c_{2} d_{1} \equiv b_{1} a_{5}$. Ma $\left(C^{\prime \prime}\right)$ deve certamente avere due piani comuni con una delle tre coppie di $T^{\prime}$, perchè, se i piani $c_{1}$, $c_{2}, c_{3}, d_{1}, d_{2}, d_{3}$ non entrassero in $T^{\prime}$, arrebbero ciascuno una retta comune con ogni coppia di $T^{\prime}$ (cfr. n..$^{\circ} 4$ ), cioè almeno tre rette di $\left(C^{\prime \prime}\right)$ apparterrebbero ad una tal coppia, il che non può essere (cfr. n. ${ }^{\circ} 3$ ).

Sicchè ogni coppia di $T^{\prime}$ è associata ad una coppia di $T^{\prime}{ }_{1}$; due coppie associate hanno due piani comuni; due coppie non associate non hanno alcun piano comune.

Può quindi $T_{1}^{\prime}$ indicarsi, ad es., cosi :

$$
T_{1}^{\prime} \equiv\left|\begin{array}{ll|ll|ll}
a_{1} & b_{1} & \alpha_{4} & b_{4} & a_{7} & b_{7} \\
\alpha_{2} & \beta_{2} & \alpha_{5} & \beta_{5} & \alpha_{8} & \beta_{8} \\
\alpha_{3} & \beta_{3} & \alpha_{6} & \beta_{6} & \alpha_{0} & \beta_{3}
\end{array}\right|:
$$


essendo $\alpha_{2}, \alpha_{3} \ldots \beta_{2}, \beta_{3} \ldots$ piani distinti dai piani $a_{2}, a_{3} \ldots b_{2}, b_{3} \ldots$ Nelle due terne $T^{\prime}, T^{\prime}{ }_{1}$, il triedro dato dai piani $a_{1}, a_{4}$ deve essere completato (cfr. n. ${ }^{0} 4$ ) da uno dei piani delle terze coppie delle due terne e per conseguenza da uno dei piani comuni a queste terze coppie, per es. da $a_{7}$ (in accordo colla notazione del n. ${ }^{\circ} 4$ ). Si ha quindi il triedro $a_{1} a_{4} a_{7}$ e parimenti i triedri $a_{1} b_{4} b_{i}$, $b_{1} a_{4} b_{7}, b_{1} b_{4} a_{7}$.

Adunque:

Rispetto ad una terna $T$, le altre 39 si dividono in due gruppi di 12 e 27. Ciascuna terna del $1^{\circ}$ gruppo ha comune colla terna considerata un enneaedro di $1^{a}$ specie: e ciascuna del $2^{\circ}$ gruppo ha colla stessa comuni sei piani che si possono distribuire in quattro triedri.

Per due terne che hanno comune un enneaedro di $1^{a}$ specie, ogni coppia dell'una ha un piaño comune (e uno solo) con ogni coppia dell' altra. Per due terne aventi a comune sei piani, ogni coppia dell'una è associata ad una coppia dell'altra, due coppie associate avendo due piani comuni. Inoltre, in questo secondo caso, le tre rette d'intersezione delle tre paja di piani (due piani di un pajo essendo comuni a due coppie associate) esistono in uno stesso piano: cioè, mantenendo le indicazioni superiori, le tre rette $a_{1} b_{1}, a_{1} b_{4}, a_{7} b_{7}$ a due a due s'incontrano. Perocchè, ad es., i due triedri $a_{1} a_{4} a_{7}, a_{1} b_{4} b_{7}$ appartengono a due coppie che hanno comuni cinque rette, le tre di $a_{1}$ e le $a_{4} b_{4}, a_{7} b_{7}$. Queste ultime (cfr. n. ${ }^{\circ}$ ) debbono adunque giacere in un piano.

9. Continuando a considerare il $2^{\circ}$ caso surricordato, non possono tre paja di piani $a_{1}, b_{1} ; a_{4}, b_{4} ; a_{7}, b_{7}$ essere comuni a tre o più terne $T$ (ogni pajo appartenendo a due coppie associate), perchè, ognuno de' quattro triedri che si possono formare con quei sei piani entrerebbe allora in più di due enneaedri (della stessa specie), il che non è possibile (n. ${ }^{\circ}$ ).

Ciò premesso, se $a_{i}, b_{1}$ sono due piani qualunque che hanno a comune una retta (della superficie), le quattro terne $T^{\prime}, T^{\prime}{ }_{1}, T^{\prime}{ }_{2}, T_{3}^{\prime}$, date dalle quattro coppie di triedri a cui appartengono $a_{1}, b_{1}$, saranno, prese a due a due, nel $2^{\circ}$ caso menzionato, ciò̀ avranno sei piani comuni: ad es.:

$$
\begin{aligned}
& T^{\prime}, \quad T_{1}^{\prime}, \quad \text { i piani } a_{1}, b_{1} ; a_{4}, b_{4} ; a_{7}, b_{7}, \\
& T_{2}^{\prime}, \quad T_{3}^{\prime}, \quad " a_{4}, b_{1} ; \quad a_{4}^{\prime}, b_{4}^{\prime} ; a_{7}^{\prime}, b_{7}^{\prime} \text {, } \\
& \ldots \ldots \ldots \ldots \ldots \ldots \ldots \ldots
\end{aligned}
$$

Due qualunque di questi sei sistemi di sei piani non hanno a comune che $a_{1}, b_{1}$. Poichè, se fosse $a_{4} \equiv a_{4}^{\prime}$ (ad es.), dall' esistenza del triedro $a_{1} a_{4} a_{7}$ e del triedro

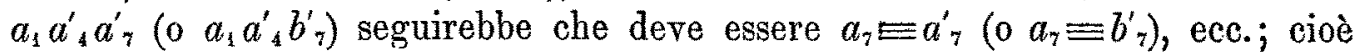
si avrebbero più di due terne $T$ colle stesse tre paja comuni. 


\section{$\S 4$. Ordinamento e costruzione delle terne $T$.}

10. Sia

$$
a_{1} a_{2} a_{3} a_{4} a_{5} a_{6} a_{7} a_{8} a_{9}
$$

un enneaedro di $1^{\text {a }}$ specie e sieno $T^{\mathrm{I}}, T^{\mathrm{H}}, T^{\mathrm{H}}, T^{\mathrm{rv}}$ le quattro terne $T$ a cui appartiene. Rispetto ad una di esse $T^{\prime \prime}$, consideriamo i due gruppi contenenti rispettivamente 12 e 27 terne $T$, di cui si disse nel $n .^{\circ}$ 8: al primo dei quali

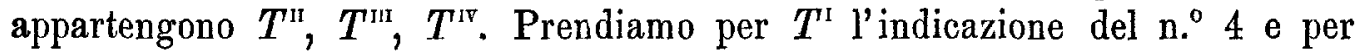
una terna del gruppo delle 27 l'indicazione del $n .^{\circ}$ 8. Poichè $a_{1} a_{4} a_{7}$ è un triedro, l' enneaedro

$$
a_{1} \alpha_{2} \alpha_{3} \alpha_{4} \alpha_{5} \alpha_{6} a_{7} \alpha_{8} \alpha_{9}
$$

è di $1^{\text {a }}$ specie. Tale enneaedro, avendo con $\left(E_{1}\right)$ un triedro comune, apparterrà (n. ${ }^{\circ}$ ) ad una delle terne $T^{\text {II }}, T^{\prime \prime \prime}, T^{\prime v}$. Adunque, mentre ognuna delle 12 terne ha con $T^{1}$ un enneaedro comune, cadauna delle altre 27 ha un enneaedro comune con qualcuna delle $T^{\mathrm{II}}, T^{\mathrm{III}}, T^{\mathrm{iv}}$.

Segue che le 40 terne $T$ possono essere ordinate, partendo da un enneaedro di $1^{\mathrm{a}}$ specie, nel modo seguente. L'enneaedro è comune a quattro terne: ognuna di queste contiene tre nuovi enneaedri di $1^{\alpha}$ specie e ciascuno di tali enneaedri appartiene a tre nuove terne. Si ottengono cosi le $40(=4+4 \cdot 3 \cdot 3)$ terne $T$.

In relazione a tale ordinamento, essendo $T^{\mathrm{r}}, T^{\mathrm{\prime}}, T^{\mathrm{II}}, T^{. \mathrm{v}}$ le quattro terne in cui entra l'enneaedro $\left(E_{1}\right)$, indicheremo con $T_{i j}^{l e}(k=\mathrm{I}$, II, III, Iv) una terna che ha comune con $T^{k}$ uno stesso enneaedro [differente da $\left(E_{1}\right)$ ], convenendo che per le terne che hanno con $T^{k}$ lo stesso enneaedro comune si tenga fisso l'indice $i$ e si varii l'indice $j$ (onde $i=1,2,3 ; j=1,2,3$ ).

11. Coll'ajuto delle proprietà precedenti, si ottiene il seguente specchio delle 40 terne $T\left({ }^{*}\right)$ :

(") Per passare alla notazione Jordar-Creniona, basta porre (ad es.) le seguenti egua. glanze:

$$
\begin{array}{lllll}
1=c_{1} & 10=c_{9} & 19=d_{7} & 28=d_{8} & 37=e_{6} \\
2=e_{2} & 11=a_{8} & 20=b_{3} & 29=b_{8} & 38=d_{4} \\
3=b_{6} & 12=c_{6} & 21=b_{5} & 30=c_{8} & 39=b_{7} \\
4=b_{9} & 13=a_{2} & 22=a_{9} & 31=e_{4} & 40=a_{7} \\
5=e_{1} & 14=c_{4} & 23=e_{8} & 32=a_{6} & 41=b_{1} \\
6=c_{2} & 15=a_{5} & 24=c_{5} & 33=e_{3} & 42=b_{4} \\
7=d_{2} & 16=c_{7} & 25=d_{3} & 34=d_{9} & 43=e_{9} \\
8=a_{3} & 17=c_{3} & 26=b_{2} & 35=a_{4} & 44=e_{7} \\
9=d_{1} & 18=e_{5} & 27=d_{5} & 36=d_{6} & 45=a_{1}
\end{array}
$$

[Cfr. Cremona, 1. c. (nei Rendiconti del R. Istituto Lombardo), pag. 209]. 


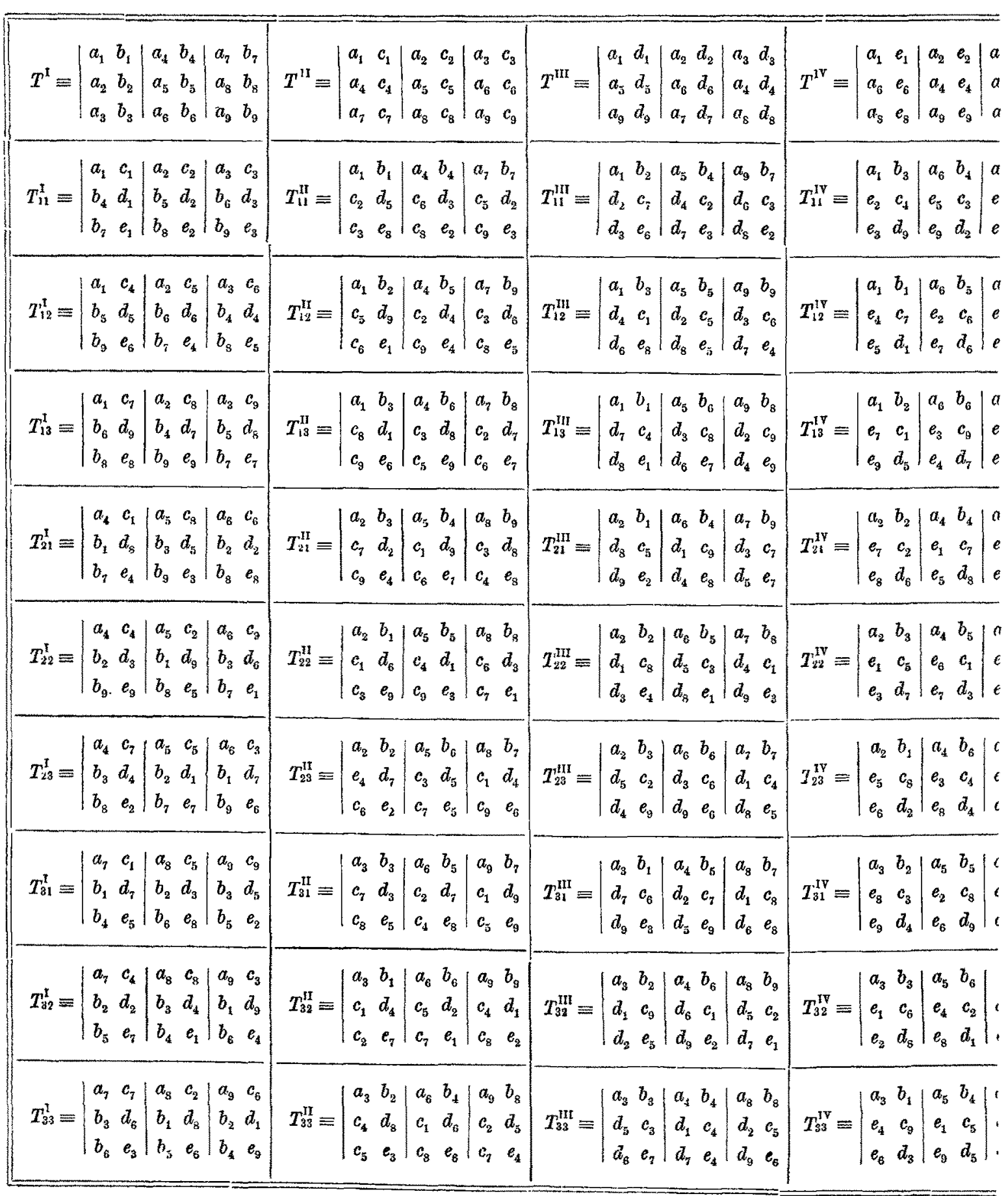


Le terne $T^{\mathrm{I}}, T^{\mathrm{II}}, T^{\mathrm{nr}}, T^{\mathrm{r}}$, nelle quali entra l'enneaedro

$$
a_{1} a_{2} a_{3} a_{4} a_{5} a_{6} a_{7} a_{8} a_{9}
$$

si scrivono immediatamente tenendo presente il determinante del $n .^{\circ} 4$ e aggiungendo $i$ nuovi simboli $b_{1}, \ldots b_{9}, c_{1}, \ldots c_{9}, d_{1}, \ldots d_{9}, e_{1}, \ldots e_{9}$, che, coi precedenti $a_{1}, \ldots a_{9}$, rappresentano tutti i 45 piani tritangenti (cfr. n. ${ }^{\circ} 5$ ). 亡 evidente che la successione di questi trentasei nuovi simboli non è interamente fissata, potendosi scambiare, in modo affatto arbitrario, quelli che figurano in una stessa colonna delle $T^{k}(k=\mathrm{I}$, II, III, IV $)$.

Si consideri l'enneaedro (di $1^{a}$ specie) $a_{1} a_{2} a_{3} b_{4} b_{5} b_{6} b_{7} b_{8} b_{9}$. Per l'arbitrarietà ora avvertita, si può ritenere che sieno triedri $a_{1} b_{4} b_{7}, a_{1} b_{5} b_{9}, a_{1} b_{6} b_{8}$ e allora che sia un triedro anche $a_{2} b_{4} b_{7}$, poichè, se si avesse invece $a_{2} b_{4} b_{8}$, si scambierebbe $b_{8}$ con $b_{9}, b_{6}$ con $b_{5}$. Quindi, per il ragionamento fatto nel n. ${ }^{\circ} 4, \mathrm{i}$ 12 triedri dell' enneaedro suddetto sono dati dalle orizzontali, dalle verticali, dai termini positivi e dai negativi del determinante

$$
\begin{array}{lll}
a_{1} & b_{4} & b_{7} \\
a_{2} & b_{5} & b_{8} \\
a_{3} & b_{6} & b_{3}
\end{array} \mid
$$

Ciò permette di scrivere la $1^{\mathrm{a}}, 3^{\mathrm{a}}, 5^{\mathrm{a}}$ colonna delle $T_{11}^{\mathrm{i}}, T_{12}^{\mathrm{r}}, T_{13}^{\mathrm{1}}$ aventi quell'enneaedro comune con $T^{\prime}$. Per ottenere le altre colonne delle nominate tre terne, osservisi che esse (all' infuori dell' enneaedro considerato) nulla hanno a comune fra loro (n. ${ }^{\circ} 5$ ), mentre ciascuna arrà con ciascuna delle $T^{\mathrm{H}}, T^{\mathrm{nl}}, T^{\mathrm{tv}}$ sei piani comuni (n..$^{\circ}$ ), giacchè (ad es.) $T_{11}^{\mathrm{L}}$ e $T^{\mathrm{nI}}$ contengono amendue $a_{1} \mathrm{e}$ non $b_{4}, b_{7}$. Approfittando quindi della sunnotata arbitrarietà, si possono scrivere le colonne mancanti: cioè (ad es.) $T_{11}^{\mathrm{s}}$ deve contenere nella seconda colonna (pel confronto con $T^{\mathrm{r}}$ ) uno dei simboli $c_{1}, c_{4}, c_{7}$ : si è posto $c_{1}$, e allora $T_{12}^{l}$ deve contenere, pure nella seconda colonna, una delle $c_{4}, c_{7}$ : posto $c_{4}$, nella seconda colonna di $T_{13}^{\mathrm{t}}$ deve trovarsi $c_{7}$, ecc.

Proponiamoci ora di scrivere $T_{11}^{\mathrm{II}}, T_{12}^{\mathrm{HI}}, T_{13}^{\mathrm{HI}}$, aventi a comune con $T^{\mathrm{II}} l^{\prime}$ enneaedro $a_{1} a_{4} a_{7} c_{2} c_{5} c_{8} c_{3} c_{6} c_{9}$. I tre simboli $a_{1}, a_{4}, a_{7}$ si troveranno distribuiti nelle tre coppie di ciascuna di quelle terne: e, siccome dal confronto di $T_{11}^{1}$, $T_{12}^{1}, T_{13}^{1}$ con $T^{11}$ segue $\left(\mathrm{n} .^{\circ} 8\right)$ rispettivamente che $a_{1} c_{2} c_{3}, a_{1} c_{5} c_{6}, a_{1} c_{8} c_{9}$ sono tre triedri, si hanno subito le prime colonne di quelle tre terne. Inoltre, dorendo ognuna delle stesse terne avere con $T^{\prime}$ sei piani comuni (giacchè le terne colle quali $T^{\mathrm{H}}$ ha enneaedri comuni sono le $T^{\mathrm{H}}, T^{\mu}, T^{\mathrm{i}}, T_{i j}^{i}$ ) ed essendo ancora arbitraria la successione dei simboli $b_{1}, b_{2}, b_{3}$, finora non adoperati, possiamo fissare che $b_{1}$ sia in $T_{11}^{\mathrm{uI}}, b_{2}$ in $T_{12}^{\mathrm{II}}, b_{3}$ in $T_{13}^{\mathrm{ul}}$. 
Ciò fatto, osservisi che $T_{11}^{\mathrm{II}}$ deve avere $\left(\mathrm{n}^{\circ} 8\right)$ un enneaedro a comune con $T_{11}^{\mathrm{r}}\left(a_{1}, c_{2}, c_{3}\right.$ essendo distribuiti nelle tre coppie di quella terna) e questo enneaedro deve essere $a_{1} b_{4} b_{7} c_{2} d_{2} t_{3} d_{3} e_{3}$. Adunque la quarta e sesta colonna di $I_{11}^{\text {II }}$ conterranno $\mathrm{i}$ simboli $b_{4}, b_{7}, d_{2}, d_{3}, e_{2}, e_{3}$. Ora $T_{11}^{\text {II }}$ deve avere sei piani comuni non solo con $T_{1}$, ma altresì con $T^{\mathrm{II}}, T^{\mathrm{IV}}\left(\mathrm{n}^{\circ}{ }^{\circ} 8\right.$ ): cioè la quarta colonna di quella terna deve essere formata di tre simboli presi rispettivamente dalle tre colonne quarta, sesta e quarta di $T^{\mathrm{I}}, T^{\mathrm{ni}}, T^{\mathrm{iv}}$ : e però la detta quarta colonna deve essere necessariamente $b_{4} d_{3} e_{2}$. Analogamente si trova la sesta colonna di $T_{11}^{\mathrm{II}}$, cioè $b_{7} d_{2} e_{3}$. Nella terza e quinta colonna di $T_{11}^{\mathrm{II}}$ debbono esserci $c_{5}, c_{6}, c_{8}, c_{9}$ (essendo $a_{4} a_{4} a_{7} c_{2} c_{5} c_{8} c_{3} c_{6} c_{9}$ l'enneaedro comune a $T_{11}^{\mathrm{HI}}, T^{\mathrm{ul}}$ ): ma $T_{11}^{\mathrm{HI}}$ deve avere tanto con $T_{42}^{\mathrm{\prime}}$ che con $T_{13}^{\mathrm{L}}$ sei piani comuni, e cio dà immediatamente le nominate colonne. Similmente si ottengono le ultime quattro colonne di $T_{12}^{\mathrm{rI}}, T_{13}^{\mathrm{HI}}$.

Per completare la seconda colonna di $T_{11}^{\mathrm{II}}$, si noti che, dovendo avere questa terna sei piani comuni con $T^{\mathrm{HI}}, T^{\mathrm{rv}}, T^{\mathrm{1}}{ }_{22}, T^{\mathrm{r}}{ }^{3}$, in detta colonna debbono entrare tre simboli tali che uno (e uno solo) appartenga ad ognuna delle quattro colonne

$$
\begin{array}{llll}
d_{1} & e_{1} & c_{4} & c_{7} \\
d_{5} & e_{6} & d_{5} & d_{9} \\
d_{9} & e_{8} & e_{8} & e_{8} .
\end{array}
$$

Ciò darebbe due soluzioni: l'una $b_{1} d_{5} e_{8}$, l'altra $b_{1} d_{9} e_{6}$. Quest'ultima è da escludere, perchè conduce ad un assurdo. In vero, ammettendola, si ottiene l'enneaedro $b_{1} d_{9} e_{6} b_{4} d_{3} e_{2} b_{7} d_{2} e_{3}$, che ha coll' altro $b_{1} b_{2} b_{3} a_{4} a_{5} a_{6} b_{7} b_{8} b_{9}$ (nascente da $T^{\prime)}$ il triedro comune $a_{4} b_{1} b_{7}$. Questi due enneaedri debbono adunque appartenere ad una stessa terna $\tau\left(\right.$ n. $\left.^{\circ} 7\right)$; della quale un triedro di una coppia è appunto $a_{4} b_{4} b_{7} \mathrm{e}$, per le due coppie rimanenti, due triedri contengono $a_{5}, a_{6}$, $b_{2}, b_{3}, b_{8}, b_{9}$ e $\mathrm{i}$ due conjugati contengono $c_{6}, c_{8}, d_{2}, d_{9}, e_{3}, e_{6}$. Il paragone di $\tau$ con $T^{\text {III }}$ e $T_{13}^{\mathrm{I}}$ mostra che $b_{8}, a_{5}$ devono trovarsi con $d_{2}$ in una stessa coppia di $\tau$ e appartenere ad un triedro. Il quale non può essere altro che $a_{5} b_{8} b_{2}$ (considerando la terna $T^{\mathrm{L}}$ e osservando che, per questa terna e per la $T_{13}^{\mathrm{HI}}, b_{3} a_{4} b_{8}$ è un triedro). Infine, confrontando $\tau$ con $T^{\mathrm{u}}, T^{\mathrm{uv}}$, si trova che $a_{5}$ deve essere nella stessa coppia con $c_{8}, e_{3}$, cioè che il triedro conjugato ad $a_{5} b_{8} b_{8}$ è $d_{9} e_{3} r_{8}$. Adunque due coppie di $\tau$ sono

$$
\begin{array}{ll}
a_{5} & c_{8} \\
b_{2} & d_{9} \\
b_{8} & e_{3}
\end{array}|, \quad| \begin{array}{ll}
a_{6} & c_{6} \\
b_{3} & d_{2} \\
b_{9} & e_{6}
\end{array} \mid .
$$


Il che è assurdo, come risulta facilmente dal confronto di t con $T_{11}^{1}\left(\mathrm{n}^{\circ}{ }^{\circ}\right.$ ). La seconda colonna di $T_{11}^{\mathrm{II}}$ è adunque $b_{1} d_{5} e_{8}$.

Delle due soluzioni $b_{2} d_{9} e_{1}, b_{2} d_{1} e_{8}$, che pure si presentano per la seconda colonna di $T_{12}^{\text {II }}$, si elimina ora facilmente la seconda $b_{2} d_{1} e_{8}$, per l'osservazione che $T_{11}^{\mathrm{II}}, T_{12}^{\mathrm{II}}$ non possono avere $\left(\mathrm{n}^{\circ} 5\right.$ ) altri piani comuni, all'infuori di quelli dell' enneaedro $a_{1} a_{4} a_{7} c_{2} c_{5} c_{8} c_{3} c_{6} c_{9}$. E parimenti si trova la seconda colonna $b_{3} d_{1} e_{6}$ di $T_{13}^{\mathrm{II}}$.

Dopo ciò, la determinazione delle altre terne si fà senza difficoltà. Per es., considerando $T_{11}^{\mathrm{III}}$ (che ha comune con $T^{\mathrm{II}}, T_{12}^{\mathrm{II}}, T_{13}^{\mathrm{mI}}$ lo stesso enneaedro $\left.a_{1} a_{5} a_{9} d_{2} d_{6} d_{7} d_{3} d_{4} d_{8}\right)$, se ne ottengono la prima e le ultime quattro colonne, come per $T_{11}^{\mathrm{II}}$. Per trovare la seconda colonna si paragoni $T_{11}^{\mathrm{HI}}$ con $T^{\mathrm{I}}, T^{\mathrm{II}}$, $T^{\mathrm{rv}}, T_{12}^{\mathrm{x}}, T_{13}^{\mathrm{1}}, T_{12}^{\mathrm{n}}, T_{13}^{\mathrm{II}}$, [con ciascuna delle quali, oltre $\alpha_{1}$, deve avere comune (n. ${ }^{\circ}$ ) altri cinque piani]: e ne segue che dei tre simboli di quella colonna uno (e uno solo) deve figurare in ciascuna delle colonne:

$$
\begin{array}{lllllll}
b_{1} & c_{1} & e_{1} & c_{4} & c_{7} & b_{2} & b_{3} \\
b_{2} & c_{4} & e_{6} & d_{5} & d_{9} & d_{9} & d_{1} \\
b_{3} & c_{7} & e_{8} & e_{6} & e_{8} & e_{1} & e_{6} ;
\end{array}
$$

ciò che dà l'unica soluzione $b_{2} c_{7} e_{6}$. Similmente si ottengono $T_{12}^{\mathrm{rrr}}, T_{13}^{\mathrm{III}}, T_{11}^{\mathrm{rv}}$, $T_{12}^{\mathrm{V}}, T_{13}^{\mathrm{N}}$.

Vogliasi ancora determinare $T_{21}^{\mathrm{r}}$ che, insieme a $T_{22}^{\mathrm{x}}, T_{23}^{\mathrm{r}}$, ha comune con $T^{\mathrm{L}}$ l'enneaedro $b_{1} b_{2} b_{3} a_{4} a_{5} a_{6} b_{7} b_{8} b_{9}$. Pel confronto di $T^{\mathrm{I}}$ con $T_{1 j}^{k_{j}}(k=\mathrm{II}, \mathrm{III}$, Iv; $j=1,2,3$ ), si hanno i triedri $a_{4} b_{1} b_{7}, a_{4} b_{2} b_{9}, a_{4} b_{3} b_{8}, a_{5} b_{2} b_{7}, a_{5} b_{3} b_{9}, \ldots$ : onde si ottengono la prima, terza e quinta colonna di $T_{21}^{\mathrm{T}}$ (e di $T_{22}^{1}, T_{23}^{\mathrm{r}}$ ). Poi, paragonando $T_{21}^{\mathrm{r}}$ con $T^{\mathrm{II}}, T^{\mathrm{HI}}, T^{\mathrm{Iq}}$, nelle quali c'̀ $a_{4}$ e con $T_{11}^{\mathrm{I}}, T_{12}^{\mathrm{r}}$, $T_{13}^{\mathrm{l}}$, nelle quali trovasi $b_{7}$, si hanno le sei colonne

$$
\begin{array}{llllll}
c_{1} & d_{3} & e_{2} & c_{9} & c_{5} & c_{1} \\
c_{4} & d_{4} & e_{4} & d_{8} & d_{6} & d_{1} \\
c_{8} & d_{8} & e_{9} & e_{7} & e_{4} & e_{1} ;
\end{array}
$$

dalle quali nasce l'unica soluzione $c_{1} d_{8} e_{4}$ per la seconda colonna di $T_{21}^{1}$. In modo analogo si trovano le altre due colonne.

Manifestamente il procedimento ora seguito vale per le terne rimanenti. 


\section{$\S 5$. Teoremi sngli enneaedri.}

12. Sia $T^{\mathrm{x}}$ una terna qualsivoglia, e sieno

$$
\begin{aligned}
& a_{1} a_{2} a_{3} a_{4} a_{5} a_{6} a_{7} a_{8} a_{9} \\
& a_{1} a_{2} a_{3} b_{4} b_{5} b_{6} b_{7} b_{8} b_{9}
\end{aligned}
$$

due enneaedri di $1^{\mathrm{a}}$ specie nascenti da essa; onde i piani $a_{1}, a_{2}, a_{3}$ comuni ai due enneaedri formano una colonna (triedro di una coppia) di $T^{\mathrm{I}}$. Un'altra terna $T^{\text {II }}$, a cui appartenga $\left(E_{1}\right)$, e un'altra $T_{\text {t1 }}^{\text {I }}$, a cui appartenga $\left(E_{1}^{\prime}\right)$, non possono avere un enneaedro a comune. Perocchè i tre piani $a_{1}, a_{2}, a_{3}$ si distribuiscono nelle tre coppie tanto di $T^{11}$ quanto di $T_{11}^{1}\left(\mathrm{n}^{\circ}{ }^{0} 8\right)$ e gli altri piani delle colonne (triedri) nelle quali entrano $a_{1}, a_{2}, a_{3}$ sono differenti, sono cioè per $T^{11}$ i piani $a_{4}, a_{5}, \ldots a_{9}$ e per $T_{11}^{\mathrm{l}}, b_{4}, b_{5}, \ldots b_{9}$. Adunque, le nuove 12 terne, nelle quali entrano i quattro enneaedri di $1^{\mathrm{a}}$ specie di $T^{\mathrm{T}}$, contengono nuovi 36 enneaedri di $1^{a}$ specie, differenti da quelli (cfr. n. ${ }^{0}$ 5) e fra loro. In altre parole, gli enneaedri di $1^{2}$ specie possono essere ordinati, a partire da una terna, nello stesso modo come le terne, a partire da un enneaedro $\left(\mathrm{n}^{\circ}{ }^{\circ} 10\right)$. Ciò̀, la terna contiene quattro enneaedri: ognuno di questi appartiene a tre nuove terne e ciascuna di tali terne contiene tre nuovi enneaedri: e cosi si hanno $i 40(=4+4 \cdot 3 \cdot 3)$ enneaedri di $1^{a}$ specie. Ad es., adottando le indicazioni dei $n .{ }^{i} 10,11$, tutti gli enneaedri di $1^{\text {a }}$ specie sono dati da uno qualunque di questi quattro gruppi di terne $(k=\mathrm{I}, \mathrm{II}, \mathrm{Inr}, \mathrm{Iv} ; i=1,2,3 ; j=1,2,3)$

$$
\left(T^{k}, T_{i j}^{\mathrm{I}}\right), \quad\left(T^{k}, T_{i j}^{\mathrm{1}}\right), \quad\left(T^{k}, T_{i j}^{\mathrm{MI}}\right), \quad\left(T^{k}, T_{i j}^{1 !}\right) .
$$

13. Due enneaedri di $1^{\prime}$ specie, appartenenti ad una medesima terna, nascono l'uno dall' altro sostituendo a due triedri i loro conjugati $\left(n .^{0} 5\right)$. Per conseguenza, in virtù della proprietà precedente $\left(\mathrm{n}^{0}{ }^{0} 12\right)$, da un enneaedro di $1^{\text {a }}$ specie provengono tutti gli altri, sostituendo successivamente a due triedri i loro conjugati. Con la stessa sostituzione si ottiene poi da un enneaedro di $2^{\mathrm{a}}$ specie un altro pure di $2^{\mathrm{a}}$ specie; mentre da un enneaedro di $1^{\mathrm{a}}\left(\mathrm{l}^{\mathrm{a}}\right)$

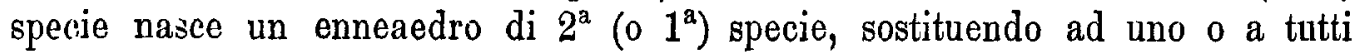
tre $i$ triedri i loro conjugati $\left(\mathrm{n}^{0}{ }^{0} 5\right)$.

Si conclude facilmente che, da un enneaedro qualsivoglia (di $1^{a}$ o $2^{a}$ specie) nascono gli altri 199, facendo successivamente l'operazione di sostituire ad un triedro il suo conjugato; due enneaedri essendo della stessa specie o di specie diversa, secondochè il numero delle dette sostituzioni è pari o dispari. 
14. Rispetto ad un ennededro di $1^{a}$ specie, gli altri 39 si dividono in due classi; l'una di 12, l'altra di 27 enneaedri. L'enneaedro considerato ha un triedro comune con ciascuno del $1^{\circ}$ gruppo e un piano comune con ciascuno del $2^{\circ}$.

Infatti se

$$
a_{1} a_{2} a_{3} a_{4} a_{5} a_{6} a_{7} a_{8} a_{9}
$$

è un enneaedro qualsiasi di $1^{\mathrm{a}}$ specie e si adottano le denominazioni del $\mathrm{n}^{0}{ }^{0} 10$, ciascuno dei dodici enneaedri esistenti [oltre $\left.\left(E_{1}\right)\right]$ in $T^{1}, T^{\mathrm{H}}, T^{\mathrm{II}}, T^{\mathrm{TV}}$, contiene manifestamente $\left(n .^{\circ} 5\right)$ un triedro di $\left(E_{1}\right)$. Questi 12 enneaedri si dividono in 4 gruppi, composto ciascuno di tre enneaedri (nascenti da una delle quattro terne suddette) e corrispondenti ad una decomposizione di $\left(E_{1}\right)$ in tre triedri, i tre enneaedri del gruppo contenendo rispettivamente questi tre triedri. Inoltre un piano di $\left(E_{1}\right)$ entra in quattro suoi trjedri e però fa parte di quatitro enneaedri appartenenti a gruppi differenti.

Gli altri 27 enneaedri nascono da uno qualunque dei quattro gruppi di nove terne $T_{i j}^{\mathrm{i}}, T_{i j}^{\mathrm{II}}, T_{i j}^{\mathrm{II}}, T_{i j}^{\mathrm{iv}}\left(\mathrm{n} .^{\circ} 12\right)$. Che ciascuno di questi enneaedri abbia con $\left(E_{1}\right)$ uno e un solo piano comune, segue dall'osservazione generale che, quando due terne hanno un enneaedro comune, ogni altro enneaedro dell'una ha un piano e uno solo comune con ogni altro enneaedro dell' altra. In vero se

$$
a_{1} a_{2} a_{3} b_{4} b_{5} b_{2} b_{7} b_{8} b_{9}
$$

è l'enneaedro comune a due terne $T^{\mathrm{r}}, T_{11}^{\mathrm{1}}$ ed $\left(E_{1}\right)$ è un altro enneaedro di $T^{3}$, i soli tre piani $a_{1}, a_{2}, a_{3}$ di $\left(E_{1}\right)$, comuni ad $\left(E_{1}^{\prime}\right)$, giacciono in $T_{11}^{1}$, giacchè le due terne hanno comuni $\left(n^{\circ} 5\right)$ i soli piani di $\left(E^{\prime}{ }_{1}\right)$. E quei tre piani $a_{i}$, $a_{2}, a_{3}$ sono distribuiti nelle tre coppie e situati in tre colonne (triedri delle coppie) della terna $T_{11}^{\mathrm{I}}$, le quali sono completate dai piani $b_{4}, b_{s}, \ldots b_{9}\left(\mathrm{n} .{ }^{0} 8\right)$. Talchè un enneaedro di $1^{\mathrm{a}}$ specie diverso da $\left(E^{\prime}\right)$ e appartenente alla detta terna $T_{11}^{\mathrm{k}}$, contenendo una sola di quelle tre colonne $\left(n .^{\circ} 5\right)$, ha comune con $\left(E_{1}\right)$ un piano ed uno solo.

E manifestamente, poichè ogni piano di $\left(E_{1}\right)$ entra in tre delle nove terne $T_{i j}^{k}$ ( $k$ essendo 1 o scono in nove gruppi, ciascun gruppo essendo costituito da tre enneaedri aventi con $\left(E_{1}\right)$ uno stesso piano comune. Questi nove gruppi si possono poi riunire a tre a tre in 12 terne, $\mathrm{i}$ tre gruppi di una terna corrispondendo ai tre piani di un triedro di $\left(E_{1}\right)$.

Un piano $a_{1}$ di $\left(E_{1}\right)$ appartiene a quattro enneaedri della $1^{a}$ classe esistenti in $T^{\mathrm{I}}, T^{\mathrm{II}}, T^{\mathrm{II}}, T^{\mathrm{IV}}$ (nelle quali terne hanno comune con $\left(E_{1}\right)$ la colonna in 
cui entra $a_{1}$ ) e che diremo $E_{1}^{\mathrm{I}}, E_{1}^{\mathrm{II}}, E_{1}^{\mathrm{III}}, E_{1}^{\mathrm{IV}}$; e a tre della $2^{\text {a }}$ classe che nascono, ad es., dalle terne $T_{11}^{\mathrm{I}}, T_{12}^{\mathrm{I}}, T_{13}^{1}$ aventi a comune con $T^{1}$ l'enneaedro $E_{1}^{\mathrm{i}}$ e che diremo $E_{11}, E_{12}, E_{13}$. Questi tre enneaedri debbono trovarsi anche nelle terne $T_{i j}^{\mathrm{n}}\left(\mathrm{n}^{\circ}{ }^{0}\right.$ 12) e precisamente in quelle tre $T_{11}^{\mathrm{n}}, T_{12}^{\mathrm{H}}, T_{13}^{\mathrm{nI}}$ che contengono $a_{1}$, cioè hanno a comune con $T^{\text {II }}$ l' enneaedro $E_{1}^{11}$ e parimenti nelle $T_{11}^{\mathrm{III}}, T_{12}^{\mathrm{III}}, T_{13}^{\mathrm{III}}$ e nelle $T_{11}^{\mathrm{IV}}, T_{12}^{\mathrm{IV}}, T_{13}^{\mathrm{Iv}}$ che hanno rispettivamente con $T^{\mathrm{III}}, T^{\mathrm{IV}}$ comuni gli enneaedri $E_{1}^{\text {II }}, E_{1}^{\text {IV }}$. Per l'osservazione generale fatta superiormente, due dei quattro enneaedri $E_{1}^{1}, E_{1}^{\mathrm{II}}, E_{1}^{\mathrm{II}}, E_{1}^{\mathrm{IV}}$ e similmente due dei quattro enneaedri $\left(E_{1}\right), E_{11}, E_{12}, E_{13}$ hanno un solo piano comune: invece ogni enneaedro della prima quaderna ha comune con ognuno della seconda un triedro, giacchè i due enneaedri appartengono ad una medesima terna [cioè $\left(E_{1}\right), E_{1}^{k}$ alla $i^{k} ; E_{1 i}, E_{1}^{k}$ alla $\left.T_{1 i}^{k}\right]$. Adunque; uno stesso piano è comune ad otto enneaedri di $1^{a}$ specie (*), distribuiti in due quaderne: due enneaedri di una stessa quaderna non hanno altro piano comune; ma ciascun cnneaedro di una quaderna ha comuni con ciascun enneaedro dell'altra due nuovi piani, che con quello formano un triedro. Il numero di queste coppie di quaderne conjugate è eguale al numero dei piani, cioè 45 .

Per le notazioni del $n .{ }^{\circ} 11$, gli otto enneaedri che hanno a comune $a_{i}$ sono

$$
\begin{aligned}
\left(E_{1}\right) & \equiv a_{1} a_{2} a_{3} a_{4} a_{5} a_{6} a_{7} a_{8} a_{9} \\
E_{11} & \equiv a_{1} b_{4} b_{7} c_{2} d_{2} e_{2} c_{3} d_{3} e_{3} \\
E_{12} & \equiv a_{1} b_{5} b_{9} c_{5} d_{5} e_{4} c_{6} d_{4} e_{5} \\
E_{13} & \equiv a_{1} b_{6} b_{8} c_{8} d_{7} e_{9} c_{9} d_{8} e_{7} \\
E_{1}^{\prime} & \equiv a_{1} a_{2} a_{3} b_{4} b_{5} b_{6} b_{7} b_{8} b_{9} \\
E_{1}^{\mathrm{II}} & \equiv a_{1} a_{1} a_{7} c_{2} c_{5} c_{8} c_{3} c_{6} c_{9} \\
E_{1}^{\mathrm{nI}} & \equiv a_{1} a_{5} a_{9} d_{2} d_{6} d_{7} d_{3} d_{4} d_{8} \\
E_{1}^{\mathrm{Iv}} & \equiv a_{1} a_{6} a_{3} e_{2} e_{4} e_{3} e_{3} e_{5} e_{7} .
\end{aligned}
$$

Le proprietà contenute in questo numero sono dovute a Cremona (**). 15. Sia

$$
b_{1} b_{2} b_{3} b_{4} b_{5} b_{6} b_{7} b_{8} b_{0}
$$

$\left(^{*}\right)$ Ciò segue anche dalla relazione $8.45=9.40$ : ovvero dall'osservare che un piano appartiene a 16 triedri e però a 16 terne 7 , e inoltre che ognuna di queste terne contiene due degli enncaedri considcrati ed ogni enneaedro appartiene a 4 delle 16 terne.

(") I. c. (nei Rendiconti del R. Istituto Lombardo), pag. 219. 
un enneaedro di $2^{a}$ specie, $T^{1}$ la terna a cui appartiene ed

$$
a_{1} a_{2} a_{3} a_{4} a_{5} a_{6} a_{7} a_{8} a_{9}
$$

l'enneaedro di $1^{\text {a }}$ specie che si ottiene, sostituendo ai tre triedri di $\left(E_{2}\right)$ i loro conjugati e che quindi esiste nella stessa terna $T^{1}$. Introducendo nuovamente le denominazioni del $n .^{0} 10$, si riconosce facilmente, con considerazioni analoghe alle precedenti, che gli altri tre enneaedri di $2^{\mathrm{a}}$ specie nascenti da $T^{\mathrm{r}}$ hanno con $\left(E_{2}\right)$ un triedro comune, mentre i quattro enneaedri di $2^{\text {a }}$ specie esistenti rispettivamente in $T^{\mathrm{II}}, T^{\mathrm{III}}, T^{\mathrm{IV}}$ non hanno con $\left(E_{2}\right)$ alcun piano comune; che quest' ultima proprietà ha luogo altresi per un enneaedro di $2^{a}$ specie di ciascuna delle terne $T_{i j}^{\mathrm{i}}$; che gli altri tre enneaedri di $2^{\mathrm{a}}$ specie provenienti da ognuna di tali terne hanno con $\left(E_{2}\right)$ quattro piani comuni; e infine che da ciascuna delle terne $T_{i j}^{\prime 1}, T_{i j}^{\prime \prime}, T_{i j}^{y}$ derivano tre enneaedri di $2^{a}$ specie aventi con $\left(E_{2}^{\prime}\right)$ un piano comune e uno con un triedro comune. Cosicchè, rispetto ad un enneaedro di $2^{a}$ specie, gli altri 159 si distribuiscono in quattro gruppi di 21, 81, 30 e 27 con nessuno, uno, tre (costituenti un triedro) $e$ quattro piani rispettivamente comuni coll'enneaedro considerato (*).

E parimenti si dimostra che, rispetto ad un enneaedro di $1^{\alpha}$ specie, $i 160$ enneaedri di $2^{a}$ specie si dividono in tre gruppi di 40,108 e 12 aventi ordinatamente nessuno, due e sei piani (formanti due triedri) comuni coll'enneaedro stesso: e che, rispetto ad un enneaedro di $2^{a}$ specie, $i 40$ enneaedri di $1^{a}$ specie si spartiscono in tre gruppi di 10, 27 e 3 aventi ordinatamente nessuno, due e sei piani (costituenti due triedri) comuni coll'enneaedro di $2^{a}$ specie.

Delle due ultime proprietà una è manifesta conseguenza dell'altra. Giacchè se $y$ è il numero degli enneaedri di $1^{a}$ specie che hanno a comune con uno di $2^{\mathfrak{a}}$ un dato numero di piani $\left(\begin{array}{llllll}0 & 0 & 2 & 0 & 6\end{array}\right)$ ed $x$ il numero degli enneaedri di $2^{a}$ specie che hanno a comune con uno di $1^{\mathrm{a}}$ lo stesso numero di piani, deve esistere la relazione

ciò̀

$$
40 \cdot x=160 \cdot y
$$

$$
x=4 y
$$

(') Nell'ultimo caso, i quattro triedri che si possono formare coi quattro piani sono di $1^{a}$ specie $\left(n .^{\circ} 16\right)$ : il che risulta dall'osservare che $\left(n .^{\circ} 18\right)$ i quattro piani comuni a lue enneaedri appartengono due ad un triedro e due ad un altro triedro dei tre ne' quali si spezza ciascuno dei due enneaedri. Ad es. due tali enneaedri sono ( $n^{\circ} 11$ )

$$
b_{1} b_{3} b_{3} b_{4} b_{5} b_{6} b_{7} b_{8} b_{9}, \quad a_{1} b_{4} b_{7} a_{2} b_{5} b_{8} c_{3} d_{3} e_{3} .
$$


§ 6. Triedri di $1^{\mathrm{a}}, 2^{\mathrm{a}}$ e $3^{\mathrm{a}}$ specie. Altre proprietà degli enneaedri.

16. I triedri considerati precedentemente sono sistemi di tre piani tritangenti che contengono nove rette della superficie esistenti in altri tre piani tritangenti. Ma è utile considerare tali sistemi di tre piani tritangenti, pei quali non abbia luogo l'ultima condizione. Si dica ancora triedr'o il sistema di tre piani tritangenti nei quali giacciono nove rette (distinte) della superficie e si chiami piano conjugato al triedro un piano che contenga una retta di ciascuno dei tre piani del triedro. Si hanno allora tre specie di triedri:

1. ${ }^{\circ}$ Triedri di $1^{a}$ specie, quelli che non ammettono piani conjugati;

2. ${ }^{\circ}$ Triedri di $2^{a}$ specie, quelli che ammettono un solo piano conjugato;

3. ${ }^{\circ}$ Triedri di $3^{a}$ specie, o Triedri di Sterner, o, come si è fatto fin qui, semplicemente Triedri (in senso stretto), quelli che hanno due e però $\left(^{*}\right.$ ) tre piani conjugati (costituenti un altro triedro della stessa specie).

I triedri di $3^{\mathrm{a}}$ specie, come si sa, sono 240, distribuiti in 120 coppie di triedri conjugati.

Per ottenere il numero dei triedri di $1^{\mathrm{a}}$ e $2^{\mathrm{a}}$ specie, si ricordi che coi 45 piani tritangenti si possono formare 720 coppie (una coppia di piani contenendo sei rette della superficie). Sieno $a_{1}, a_{2}$ i piani di una tal coppia e $a_{3}$ il piano che con essi forma un triedro di $3^{a}$ specie. Escludendo i 6 piani di questo triedro e del triedro ad esso conjugato, e i 27 che passano ulteriormente per le sue nove rette, rimangono 12 piani, ognuno de' quali costituisce, con $a_{1}$, $a_{2}$, un triedro di $1^{a}$ specie. Invece si ha un triedro di 2 specie, aggiungendo ad $a_{1}, a_{2}$, uno dei nove piani che passano per le tre rette di $a_{3}$ (all'infuori di $a_{3}$ e dei piani del triedro conjugato ad $a_{1} a_{2} a_{3}$ ). Adunque il numero dei triedri di $1^{\mathrm{a}}$ specie è $2880\left(=\frac{720 \cdot 12}{3}\right)$ e quello dei triedri di $2^{\mathrm{a}}$ specie è $2160\left(-\frac{720 \cdot 9}{3}\right)$.

17. Il sistema delle tre paja di piani (**)

$$
a_{1}, \quad b_{1} ; \quad a_{4}, \quad b_{4} ; \quad a_{7}, b_{7} ;
$$

comuni a due terne $T$, fornisce quattro triedri di $2^{3}$ specie

$$
b_{1} b_{4} b_{7}, \quad b_{1} a_{4} a_{7}, \quad a_{1} b_{4} a_{7}, \quad a_{1} a_{4} b_{7},
$$

(") Cfr., ad es, Cremona-Curtze, 1, c., n. ${ }^{\circ} 260$.

(") Si dice pajo o coppia, secondochè i due piani tritangenti hanno o no comune una rotta della superficie. 
e quattro di $3^{\mathrm{a}}$ specie

$$
a_{1} a_{4} a_{7}, \quad a_{1} b_{4} b_{7}, \quad b_{1} a_{4} b_{7}, \quad b_{1} b_{4} a_{7} ;
$$

i quali otto triedri hanno uno stesso piano conjugato, quello delle rette $a_{1} b_{1}$, $a_{4} b_{4}, a_{7} b_{7}\left(\mathrm{n} . .^{0} 8\right)$.

Ora un pajo di piani $a_{1}, b_{1}$ appartiene a sei sistemi $(\Sigma)$, ne' quali i piani rimanenti sono tutti differenti (n. ${ }^{\circ} 9$ ). Questi 24 piani sono per conseguenza i piani che non contengono rette del pajo $a_{1} b_{1}$, giacchè il numero di tali piani è appunto $45-2-4 \cdot 4-3=24$. Dato un pajo di piani $a_{1}, b_{1}$ e uno qualunque $a_{4}$ dei detti 24 piani, si ottiene il sistema $(\Sigma)$, a cui i tre piani appartengono, costruendo tre dei quattro triedri $\left(S^{\prime}\right)$. Adunque, se $i$ piani $a_{1}, b_{1}$ si segano in una retta della superficie e se $a_{4}$ è un altro piano qualsivoglia che non contenga rette di $a_{1}, a_{2}$, l'esistenza di uno qualunque dei quattro triedr $i$ $d i 3^{a}$ specie $\left(S^{\prime}\right)$ è conseguenza di quella degli altri tre.

Questa proprietà può mettersi sotto un altro aspetto. Sia dato un triedro di $2^{\text {a. }}$ specie $b_{1} b_{4} b_{7}$ e si costruiscano tre dei triedri $\left(S^{\prime}\right)$; si ottengono tre nuovi piani $a_{1}, a_{4}, a_{7}$ che, con quelli del triedro dato, formano tre paja di piani. Assunto un pajo $a_{1} b_{1}$ e uno $a_{4}$ dei quattro piani $b_{4}, b_{7}, a_{4}, a_{7}$, si ottiene un sistema $(\Sigma)$, che, in virtù de' tre triedri costruiti, deve contenere i piani rimanenti $b_{4}, b_{7}, a_{7}$. Per conseguenza (ponendo $b_{1}=a, b_{4}=b, b_{7}=c, a_{1}=a^{\prime}$, $a_{4}=b^{\prime}, b_{7}=c^{\prime}$ ) si ha il teorema:

Se $a b c$ è un triedro di $2^{a}$ specie, e se dei qualtro triedri $a b c^{\prime}, b^{\prime} c, a^{\prime} b c$, $a^{\prime} b^{\prime} c^{\prime}$ tre sono di $3^{a}$ specie, anche $i l$ quarto $\grave{e} d i 3^{a}$ specie.

In particolare, se $a b c$ è di $2^{a}$ specie e sono di $2^{2}$ specie $a b c^{\prime}, a b^{\prime} c, a^{\prime} b c$, è pure di $3^{\mathrm{a}}$ specie $a^{\prime} b^{\prime} c^{\prime}$. In questo senso si può dire che ad un triedro di $2^{a}$ specie $a b c$ corrisponde uno di terza $a^{\prime} b^{\prime} c^{\prime}$. In un sistema $(\Sigma)$ esistono quattro coppie di tali triedri corrispondenti; cioè i triedri $(S)$ sono ordinatamente corrispondenti ai triedri $\left(S^{\prime}\right)$.

Un triedro di $3^{a}$ specie corrisponde a nove triedri di $2^{2}$, determinati dai nove piani che passano per le tre rette di uno dei tre piani del triedro di $3^{a}$ specie (all'infuori del piano stesso e di quelli del triedro conjugato). La qual cosa risulta altresì dalla relazione $9 \cdot 240=2160\left(n^{\circ}{ }^{\circ} 16\right)$.

Due piani di una coppia di una terna $T$, i quali non si segano in una retta della superficie, cioè giacciono in una stessa colonna di $T$, formano con un piano qualsiasi delle altre due coppie, un triedro di $1^{a}$ specie, giacchè questo piano non ha alcuna retta comune col terzo piano della suddetta colonna. Un triedro di $2^{2}$ specie, il quale appartenga ad una terna $T$, deve adunque avere 
i suoi tre piani distribuiti nelle tre coppie di $T$. E poichè, come vedemmo testè, un triedro di $2^{\mathrm{a}}$ specie, appartiene ad un solo sistema $(\Sigma)$, si può concludere (n. ${ }^{\circ}$ ) che un triedro di $2^{a}$ specie appartiene a due sole terne $T e$ però a due soli enneaedri, che sono di $2^{a}$ specie (*).

18. Degli 84 triedri contenuti in un enneaedro di $1^{\text {a }}$ specie, 12 sono di $3^{\mathrm{a}}$ specie. I rimanenti 72 triedri sono tutti di $1^{a}$ specie. Giacchè, considerando i tre piani di un triedro di $3^{2}$ specie dell' enneaedro, uno qualsivoglia degli altri sei piani dell'enneaedro stesso, non contenendo alcuna retta di quei tre piani, forma necessariamente con due di essi un triedro di $1^{a}$ specie.

Degli 84 triedri contenuti in un enneaedro di 2 specie, 3 sono di $3^{\text {a }}$ specie. $I$ rimanenti 81 sono 54 di $1^{a}$ specie e 27 di $2^{a}$. Cioè i triedri che contengono due piani di un triedro di $3^{\mathrm{a}}$ specie sono di $1^{\mathrm{a}}$ specie e si dimostra come dianzi: mentre $\mathrm{i}$ triedri che contengono tre piani appartenenti rispettivamente ai tre triedri di $3^{a}$ specie sono di $2^{a}$ specie. In vero, se $b_{1} b_{2} b_{3} b_{4} b_{5} b_{6} b_{7} b_{8} b_{9}$ ̀̀ l'enneaedro considerato e

$$
T \equiv\left|\begin{array}{cc|cc|cc}
a_{1} & b_{1} & a_{4} & b_{4} & a_{7} & b_{7} \\
a_{2} & b_{2} & a_{5} & b_{5} & a_{8} & b_{8} \\
a_{3} & b_{3} & a_{6} & b_{6} & a_{9} & b_{9}
\end{array}\right|
$$

la terna a cui esso appartiene, cioè $b_{1} b_{2} b_{3}, b_{4} b_{5} b_{6}, b_{7} b_{8} b_{9}$ i triedri di $3^{\mathrm{a}}$ specie e $a_{1} a_{2} a_{3}, a_{4} a_{5} a_{6}, a_{7} a_{8} a_{9}$ i loro conjugati; due piani $b_{1}, b_{1}$ formano un triedro di $3^{\text {a }}$ specie con uno $a_{7}$ dei piani $a_{7}, a_{8}, a_{9}$. Ciascuno dei piani $b_{7}, b_{8}, b_{9}$ contiene una retta di $a_{7}$ e quindi $\mathrm{i}$ tre triedri $b_{1} b_{4} b_{7}, b_{1} b_{4} b_{8}, b_{1} b_{4} b_{9}$ sono di $2^{a}$ specie ed hanno rispettivamente per piani conjugati $i$ tre piani del triedro conjugato a $b_{1} b_{4} a_{7}$.

I 27 triedri di $2^{\mathrm{a}}$ specie del suddetto enneaedro hanno adunque per piani conjugati i piani dei nove triedri conjugati a nove dei 12 triedri di $3^{2}$ specie dell'enneaedro di $1^{\mathrm{a}}$ specie $b_{1} b_{2} b_{3} b_{4} b_{5} b_{6} a_{7} a_{8} a_{9}$ (o di $b_{1} b_{2} b_{3} a_{4} a_{5} a_{6} b_{7} b_{8} b_{9}$, o anche di $\left.a_{1} a_{2} a_{3} b_{4} b_{5} b_{6} b_{7} b_{8} b_{9}\right)$; escludendo cioè $\mathrm{i}$ triedri $b_{1} b_{2} b_{3}, b_{4} b_{5} b_{6}, a_{7} a_{8} a_{9}$, i cui conjugati sono $a_{1} a_{2} a_{3}, a_{4} a_{5} a_{6}, b_{7} b_{8} b_{9}$. Ne risulta, ricordando una proprietà del $n .^{\circ} 5$, che, $i 45$ piani tritangenti sono dati dai piani di un enneaedro qualsivoglia (di $1^{a}$ o $2^{a}$ specie) e dai piani conjugati ai triedri (di $2^{a}$ $e 3^{a}$ specie) contenuti in esso.

(*) Il che concorda colla relazione $\left(n \cdot{ }^{i} 16,18\right)$

$$
2160=\frac{160.27}{2}
$$


19. Vedemmo che un triedro di $3^{\text {a }}$ specie appartiene a quattro enneaedri, due di $1^{a}$ e due di $2^{a}$ specie $\left(n .^{\circ} 5\right)$ e che un triedro di $2^{a}$ specie appartiene a due enneaedri di $2^{\mathrm{a}}$ specie $\left(\mathrm{n} .^{\circ} 17\right)$.

Un triedro di $1^{a}$ specie appartiene a quattro enneaedri, uno di $1^{a}$ specie $e$ tre $d i 2^{a}$. Sia infatti $a_{1} a_{4} a_{8}$ un triedro di $1^{a}$ specie: e sia $a_{3}$ il piano che forma con $a_{4}, a_{8}$ un triedro di $3^{a}$ specie e $a_{2}$ ouello che forma pure un triedro di $3^{2}$ specie con $a_{1}, a_{3}$. Dicasi $T^{\prime}$ la terna $T$ individuata dal triedro $a_{1} a_{2} a_{3}$. Poichè $\mathrm{i}$ due triedri di $3^{\mathrm{a}}$ specie $a_{3} a_{4} a_{8}, a_{1} a_{2} a_{3}$ hanno comune $a_{3}$, e $a_{1}$ non ha retta (della superficie) comune nè con $a_{4}$ nè con $a_{8}$, lo stesso deve avvenire di $a_{2}$, perchè le due coppie a cui dànno origine quei due triedri hanno comuni le sole tre rette di $a_{3}$ (cfr. n. ${ }^{\circ}$ 3). Ciò̀ i piani $a_{4}, a_{8}$ appartengono alle due coppie di $T^{\mathrm{\prime}}$, diverse da quella in cui entra $a_{1} a_{2} a_{3}$ e appartengono a coppie differenti per essere $a_{3} a_{1} a_{8}$ un triedro di $3^{a}$ specie (cfr. n. ${ }^{\circ} 4$ ). Dopo ciò, la costruzione di $T^{1}$ non presenta alcuna difficoltà (n. $\left.{ }^{\circ} 4\right)$ : e sia (ad es.)

$$
T^{\mathrm{s}} \equiv \mid \begin{array}{ccccc|c|c|}
a_{1} & \cdot & & a_{4} & \cdot & a_{7} & \cdot \\
a_{2} & \cdot & & a_{5} & \cdot & a_{8} & \cdot \\
a_{3} & \cdot & \vdots & a_{6} & \cdot & a_{9} & \cdot
\end{array} \cdot
$$

Questa terna $T^{\prime}$ è l'unica terna $T$ a cui appartiene un triedro di $1^{a}$ specie $a_{1} a_{1} a_{8}$, se $i$ tre piani del triedro debbono appartenere uno ad uno alle tre coppie ciella terna, giacchè il piano $a_{3}$ che forma con $a_{4}, a_{8}$ un triedro di $1^{2}$ specie deve appartenere alla colonna in cui trovasi $a_{1}$ [altrimenti $a_{1} a_{4} a_{8}$ sarebbe di $2^{\imath}$ specie (cfr. n. ${ }^{\circ} 18$ )]. Ma il triedro $a_{1} a_{4} a_{8}$ appartiene ad altre tre terne $T^{\mathrm{II}}$, $T^{\mathrm{H}}, T^{\mathrm{t}}$, che si ottengono ponendo due piani del triedro dato in una stessa coppia (e in una stessa colonna) e tenendo di nuovo presente il n. $^{\circ} 4$ : le quali tre terne hanno comuni con $T^{1}$ lo stesso enneaedro di $1^{a}$ specie

$$
a_{1} a_{2} a_{3} a_{4} a_{5} a_{6} a_{7} a_{8} a_{9} .
$$

Prendendo per indicazione delle medesime quella data dallo specchio del $\mathrm{n}^{\circ} 11$, abbiamo adunque, come asserimmo, oltre il suddetto enneaedro di $1^{a}$ specie, i tre enneaedri di $2^{\mathrm{a}}$ specie

$$
\begin{aligned}
& a_{1} a_{4} a_{7} a_{2} a_{5} a_{8} c_{3} c_{6} c_{3}, \\
& a_{1} a_{5} a_{9} a_{3} a_{4} a_{8} d_{2} d_{6} d_{7} \text {, }
\end{aligned}
$$

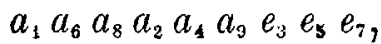

col triedro comune $a_{1} a_{1} a_{8}$.

Una conferma della precedente proprietà si ha nelle relazioni (cfr. n. ${ }^{i} 16,18$ )

$$
2880=72 \cdot 40=\frac{54 \cdot 160}{3} .
$$


20. Sia $a_{1} a_{1} a_{8}$ un triedro di $1^{\mathrm{a}}$ specie e

$$
a_{1} a_{2} a_{3} a_{4} a_{5} a_{6} a_{7} a_{8} a_{9}
$$

l'enneaedro di $1^{\mathrm{a}}$ specie individuato da esso (n. $\left.{ }^{\circ} 19\right)$. I triedri di $3^{\mathrm{a}}$ specie dati dalle coppie di piani $a_{1} a_{4}, a_{4} a_{8}, a_{4} a_{8}$ debbono essere completate da piani dell' enneaedro (n. $\left.{ }^{\circ} 4\right)$, e sieno, ad es.,

$$
a_{1} a_{4} a_{7}, \quad a_{1} a_{8} a_{6}, \quad a_{4} a_{8} a_{3} .
$$

Il triedro $a_{3} a_{6} a_{7}$ deve essere di $1^{\mathrm{a}}$ specie, perchè, se fosse di $3^{\mathrm{a}}$ specie, i quattro piani $a_{4}, a_{8}, a_{6}, a_{7}$ (ad es.) apparterrebbero ai due triedri di $3^{\mathrm{a}}$ specie $a_{1} a_{4} a_{7}$, $a_{1} a_{6} a_{8}$ con un piano comune $a_{1}$ e anche ai due triedri di $3^{4}$ specie $a_{3} a_{4} a_{8}$, $a_{3} a_{6} a_{7}$, pure con un piano comune $a_{3}$, ciò che non può essere (n. $\left.{ }^{\circ} 4\right)$. Ripetasi ora per il triedro di $1^{\mathrm{a}}$ specie $a_{3} a_{5} a_{7}$ ciò che si fece per $a_{1} a_{4} a_{8}$ : cioè si considerino i triedri di $3^{\mathrm{a}}$ specie dati dalle coppie di piani $a_{3} a_{6}, a_{3} a_{7}, a_{6} a_{7}$. Questi triedri, per l'esistenza dei due ultimi triedri $(M)$, non possono essere completati dai piani $a_{1}, a_{4}, a_{8}$ e però debbono esserlo dai piani $a_{2}, a_{5}, a_{9}$. Sieno tali triedri di $3^{\mathrm{a}}$ specie

$$
a_{3} a_{6} a_{9}, \quad a_{3} a_{7} a_{5}, \quad a_{6} a_{7} a_{2} .
$$

Questi e i triedri $(M)$ forniscono tre dei quattro triedri che hanno a comune rispettivamente $a_{3}, a_{6}, a_{7}$ : onde nasce (n. $\left.{ }^{\circ} 4\right)$ l'esistenza di questi altri triedri di $3^{\mathrm{a}}$ specie

$$
a_{3} a_{1} a_{2}, \quad a_{6} a_{5} a_{4}, \quad a_{7} a_{8} a_{9}
$$

e allora (n. ${ }^{\circ}$ ) anche dei seguenti

$$
a_{2} a_{5} a_{8}, \quad a_{2} a_{9} a_{4}, \quad a_{5} a_{3} a_{1} .
$$

Gli ultimi mostrano che, facendo per il triedro di $1^{2}$ specie $a_{2} a_{5} a_{9}$ la costruzione fatta per $a_{4} a_{4} a_{8}, a_{3} a_{6} a_{7}$, si ritorna al triedro $a_{1} a_{4} a_{8}$. Abbiamo adunque questa proprietà (ponendo $a_{1}=a, a_{4}=b, a_{8}=c, a_{3}=a^{\prime}, a_{6}=b^{\prime}, a_{7}=c^{\prime}$, $a_{2}=a^{\prime \prime}, a_{5}=b^{\prime \prime}, a_{9}=c^{\prime \prime}\left({ }^{*}\right)$ :

() Onde il determinante $\left(n *^{\circ} 4\right)$

$$
\left|\begin{array}{lll}
a_{1} & a_{2} & a_{3} \\
a_{4} & a_{5} & a_{6} \\
a_{7} & a_{8} & a_{9}
\end{array}\right|
$$

che dà i 12 triedri $(M),(N),(P),(Q)$, diventa

$$
\left|\begin{array}{lll}
a & a^{\prime \prime} & a^{\prime} \\
b & l^{\prime \prime} & b^{\prime} \\
c^{\prime} & c & c^{*}
\end{array}\right| .
$$


Se $a b c$ è un triedro di $1^{a}$ specie e sono $a b c^{\prime}, a b^{\prime} c, a^{\prime} b c, a^{\prime} b^{\prime} c^{\prime \prime}, a^{\prime} b^{\prime \prime} c^{\prime}$, $a^{\prime \prime} b^{\prime} c^{\prime}$ di $3^{a}$ specie, $i$ due triedri $a^{\prime} b^{\prime} c^{\prime}, a^{\prime \prime} b^{\prime \prime} c^{\prime \prime}$ sono di $1^{a}$ specie e $a^{\prime \prime} b^{\prime \prime} c, a^{\prime \prime} b c^{\prime \prime}$ $a b^{\prime \prime} c^{\prime \prime}$ di $3^{a}$. Inoltre sono di $3^{a}$ specie $i$ triedri $a a^{\prime} a^{\prime \prime}, b b^{\prime} b^{\prime \prime}, c c^{\prime} c^{\prime \prime}\left(^{*}\right)$.

Tali tre triedri di $1^{a}$ specie $a b c, a^{\prime} b^{\prime} c^{\prime}, a^{\prime \prime} b^{\prime \prime} c^{\prime \prime}$, uno qualunque dei quali dà origine al successivo colla costruzione dei triedri di $3^{a}$ specie dati dai suoi tre piani presi a due a due, si dirà che formano un ciclo.

I nove piani di un ciclo costituiscono un enneaedro di $1^{a}$ specie. Coi 2880 triedri di $1^{\text {a }}$ specie si formano 960 cicli. Ogni enneaedro di $1^{\mathrm{a}}$ specie contiene 24 cicli.

Si ha qui un nuovo concetto degli enneaedri di $1^{\text {a }}$ specie che, insieme alla proprietà del $n .^{\circ} 13$, potrebbe essere il punto di partenza di una trattazione degli enneaedri indipendente dalla considerazione delle terne $T$.

\section{$\S 7$. Poliedri. Pentredro principale.}

21. Nelle cose che seguono si chiama poliedro il sistema di più piani tritangenti, due qualunque dei quali si segano in una retta esterna alla superficie; ordine di un poliedro il numero dei piani che lo formano (onde in un poliedro di ordine $n$ giacciono $3 n$ rette della superficie); e poliedro principale un poliedro che non è contenuto in poliedri d'ordine superiore all'ordine di esso.

Quali poliedri principali esistono, oltre gli enneaedri di $1^{\mathrm{a}}$ e $2^{\mathrm{a}}$ specie? Si osservi anzitutto che ogni poliedro contenente un triedro di $3^{a}$ specie è necessariamente contenuto in un enneaedro. Ciò segue immediatamente dall'ultima considerazione del $n .0^{\circ} 6$. Inoltre se un poliedro (di ordine $n>3$ ) contiene un triedro di $3^{a}$ specie, contiene necessariamente anche triedri di $1^{a}$ specie; giacchè, se $a b c d .$. è un poliedro ed $a b c$ è di $3^{a}$ specie, $a b d, b c d, a c d, \ldots$ debbono essere di $1^{n}$ specie, $d$ non contenendo alcuna delle nove rette di $a b c$.

Adunque, per la risoluzione del suddetto problema, basterà considerare:

A) I poliedri che contengono almeno un triedro di $2^{\mathrm{a}}$ specie;

$B)$ I poliedri che contengono almeno un triedro di $1^{a}$ specie.

22. Prendiamo dapprima ad esaminare il caso $A$ ). Cioè abbiasi un poliedro contenente almeno un triedro di $2^{2}$ specie $a b c$. Sia $a^{\prime} b^{\prime} c^{\prime}$ il triedro di

(*) Questa proprietà vale e si dimostra identicamente per i nove flessi di una curva di $3^{o}$ ordine. Basta sostituire a piano, flesso; a triedro di $3^{a}$ specie, terna di flessi in linea retta; e a triedro di $l^{a}$ specie, terna di flessi non giacenti in linea retta (cfr. Nota al $n .^{\circ} 4$ ). 
$3^{\mathrm{a}}$ specie corrispondente ad $a b c$ (cioè, secondo $\mathrm{il} \mathrm{n}^{\circ} 17$, sieno $a b c^{\prime}, a b^{\prime} c$, $a^{\prime} b c$ triedri di $3^{\mathrm{a}}$ specie) ed $\alpha$ il piano conjugato ad $a b c$, il qual piano è pure uno dei tre piani conjugati al triedro $a^{\prime} b^{\prime} c^{\prime}$. Gli altri due piani conjugati a questo triedro dicansi $d$, e.

Ora cerchiamo i piani che non contengono rette di $a b c$. Oltre $a, b, c$, sono da escludere il piano $\alpha$ e $\mathrm{i}$ sei piani (all'infuori di $\alpha$ ) dei triedri conjugati ad $a b c^{\prime}, a b^{\prime} c, a^{\prime} b c$. Poi per ciascuna delle due rette di $a$ (e similmente di $b$ e $c$ ), non esistenti su $\alpha$, passano due nuovi piani, mentre per la retta $a \alpha$ passano tre nuovi piani. Sicchè restano

$$
45-3-1-6-2 \cdot 2 \cdot 3-3 \cdot 3=14
$$

piani che non contengono rette di $a, b, c$. Ed è facile indicare questi 14 piani: sono i due piani $d, e \theta$ i dodici piani che passano per le sei rette di $d, e$ (oltre $d, e, a^{\prime}, b^{\prime}, c^{\prime}$ e i piani conjugati ai triedri $a b c^{\prime}, a b^{\prime} c, a^{\prime} b c$ ) e che indicheremo con $f, f^{\prime}, g, g^{\prime}, h, h^{\prime}, m, m^{\prime}, n, n^{\prime}, p, p^{\prime}$.

Si facciano tre casi, cioè:

1. Si aggiungano ad $a b c$ amendue $\mathrm{i}$ piani $d, e$;

2. ${ }^{\circ}$ Si aggiunga ad $a b c$ un solo dei piani $d, e$;

$3 .^{\circ}$ Non si aggiunga ad $a b c$ alcuno dei piani $d, e$.

23. Nel $1^{\circ}$ caso si ha un pentaedro $a b c d e$, che è un poliedro principale, i dodici piani $f, f^{\prime} \ldots$ avendo rette comuni con $d$, e. I dieci triedri contenuti in questo pentaedro sono tutti di $2^{a}$ specie. Infatti $a b d$ (e parimenti $a b e$, $a c d, a c e, b c d, b c e)$ è di $2^{a}$ specie, perchè $a b c^{\prime}$ è di $3^{a}$ specie e $d$, essendo conjugato ad $a^{\prime} b^{\prime} c^{\prime}$, passa per una retta di $c^{\prime}$ : e il triedro ade (e nello stesso modo $b d e, c d e)$ è pure di $2^{\mathrm{a}}$ specie, perchè il triedro $\alpha d e$, conjugato ad $a^{\prime} b^{\prime} c^{\prime}$, è di $3^{\mathrm{a}}$ specie ed $a$ contiene una retta di $\alpha$, piano conjugato ai triedri $a b c^{\prime}$, $a b^{\prime} c$.

Viceversa: un poliedro principale ab cd..., di cui ogni triedro è di $2^{a}$ specie, deve essere un pentaedro. Poichè, considerando un suo triedro (di $2^{\mathrm{a}}$ specie) $a b c$ e ponendo le stesse denominazioni di dianzi, il piano conjugato al triedro di $2^{a}$ specie $a b d$ sarà pure conjugato al triedro di $3^{a}$ specie $a b c^{\prime}$ e quindi $d$. passerà per una retta di $c^{\prime}$; e analogamente $d$ passerà per una retta $b^{\prime}$ e per una di $a^{\prime}$ : cioè il piano $d$ sarà uno dei piani conjugati al triedro $a^{\prime} b^{\prime} c^{\prime}$ (all' infuori di $\alpha$ ): e però, ecc.

Il numero di questi pentaedri principali è $\frac{2160}{10}=216$, ognuno essendo individuato da un triedro di $2^{a}$ specie e in ognuno essendo dieci tali triedri. 
Dato un pentaedro principale, si hanno 15 rette, quelle giacenti nei cinque piani di esso e 15 piani, cioè questi cinque piani e i dieci conjugati ai dieci triedri del pentaedro. Ognuno dei 15 piani contiene tre delle 15 rette; onde per ciascuna delle 15 rette passano tre dei 15 piani: il che risulta anche dall'osservare che un piano del pentaedro appartiene a sei dei dieci triedri in esso contenuti. Adunque, per una nota proprietà (*), le 12 rette rimanenti costituiscono una bissestupla.

Reciprocamente: le 15 rette che si ottengono, togliendo dalle 27 rette le 12 di una bissestupla, esistono in sei pentaedri principali, cioè possono essere in sei modi considerate come intersezione della superficie e di cinque piani $\left(^{* *}\right)$. $I 15$ piani contenenti le 15 rette sono $i 5$ piani di un pentaedro e $i 10$ piani conjugati ai dieci triedri di esso.

Sicchè $i 216$ pentaedri principali si distribuiscono in 36 gruppi corrispondenti alle 36 bissestuple.

24. Discende dalle cose precedenti che un tetraedro, $\mathrm{i}$ cui quattro triedri sono di $2^{\mathrm{a}}$ specie, è contenuto in un pentaedro principale. Adunque il numero di quei tetraedri ̀̀ $216 \cdot 5=1080$.

\section{§. Nttaedro principale.}

25. Si esamini ora il $2^{\circ}$ caso $\left(n^{\circ} 22\right)$ : cjoè (n. ${ }^{\circ}$ 23) si consideri un poliedro il quale contenga un tetraedro appartenente ad un pentaedro principale, tale cioè che $i$ suoi quattro triedri sieno di $2^{a}$ specie. Per agevolare la discus. sione adoperiamo la notazione di SchlaEfl. Il triedro formato dai piani

$$
\begin{aligned}
& a \equiv c_{14} c_{26} c_{35}, \\
& b \equiv c_{15} c_{24} c_{36}, \\
& c \equiv c_{16} c_{23} c_{45},
\end{aligned}
$$

¿̀ di $2^{\mathrm{a}}$ specie e può concepirsi che sia uno qualsivoglia di $2^{\mathrm{a}}$ specie, intendendo che $\mathrm{i}$ numeri $1,2, \ldots 6$ rappresentino sei simboli $i_{1}, i_{2}, \ldots i_{6}$ e questi una permutazione qualunque dei numeri precedenti (con che si hanno i 60 triedri di $2^{a}$ specie contenuti nel sistema che nasce dall' escludere le 12 rette di una bissestupla) e inoltre che sia arbitraria la bissestupla di riferimento.

(') Cfr. Cremona, Teoremi stereometrici dai quali si deducono le proprietà dell'esagranmo di Pascal (Memorie della R. Accarl dei Lincei, Serie III, vol. $1^{\circ}$ ), n. 41 e s.r.i.

(") Cremona, 1. c., n. 11. 
Si ottengono immediatamente i piani

$$
\begin{aligned}
& \alpha \equiv c_{35} c_{24} c_{16}, \\
& a^{\prime} \equiv c_{46} c_{35} c_{12}, \\
& b^{\prime} \equiv c_{56} c_{13} c_{24}, \\
& c^{\prime} \equiv c_{25} c_{34} c_{16},
\end{aligned}
$$

e i quattordici piani che non contengono rette di $a b c$,

$$
\begin{aligned}
& d \equiv c_{46} c_{13} c_{25} \mid \begin{array}{ll}
f \equiv a_{4} b_{6} c_{46}, & f^{\prime} \equiv a_{6} b_{1} c_{46} \\
g \equiv a_{1} b_{3} c_{13}, & g^{\prime} \equiv a_{3} b_{1} c_{13}, \\
h \equiv a_{2} b_{5} c_{25}, & h^{\prime} \equiv a_{5} b_{2} c_{25}
\end{array} \\
& e \equiv c_{12} c_{88} c_{34} \mid \begin{array}{ll}
m \equiv a_{1} b_{2} c_{12}, & m^{\prime} \equiv a_{2} b_{1} c_{12}, \\
n \equiv a_{5} b_{6} c_{58}, & n^{\prime} \equiv a_{8} b_{5} c_{58}, \\
p \equiv a_{3} b_{4} c_{34}, & p^{\prime} \equiv a_{4} b_{3} c_{34}
\end{array}
\end{aligned}
$$

Si considerino adunque i poliedri a cui appartiene il tetraedro $a b c d$ (ad es.). In tali poliedri non entra $e$ (caso già considerato nel n. ${ }^{\circ} 23$ ), nè entrano $f, f^{\prime}$, $g, g^{\prime}, h, h^{\prime}$, i quali piani hanno ciascuno comune una retta con $d$ : e possono evidentemente figurarvi tre soli dei sei piani $m, m^{\prime}, n, n^{\prime}, p, p^{\prime}$. Onde si hanno otto poliedri principali, che sono ettaedri. Questi otto ettaedri sono (*)

$$
\begin{array}{ll}
c \cdot a b d \cdot n m p, & c \cdot a b d \cdot n^{\prime} m^{\prime} p^{\prime}, \\
b \cdot a c d \cdot p m^{\prime} n, & b \cdot a c d \cdot p^{\prime} m n^{\prime}, \\
a \cdot c b d \cdot n^{\prime} p m, & a \cdot c b d \cdot n p^{\prime} m^{\prime}, \\
d \cdot a b c \cdot m n p^{\prime}, & d \cdot a b c \cdot m^{\prime} n^{\prime} p,
\end{array}
$$

$e$ non sono essenzialmente diversi, giacchè dai quattro di sinistra si passa ai quattro di destra collo scambio delle $a_{i}, b_{i} ;$ e si passa dal primo al secondo, terzo e quarto di sinistra, scambiando rispettivamente

$$
\begin{array}{llllll}
1, & 2 ; & 3, & 5 ; & 4, & 6: \\
1, & 3 ; & 2, & 4 ; & 5, & 6: \\
1, & 5 ; & 2, & 6 ; & 3, & 4 .
\end{array}
$$

() La divisione di ciascun ettaedro in un piano e due terne è in relazione colla seguente proprietà (n. 26), essendo corrispondenti nelle due torne due piani che vi occupano lo stesso posto. 
26. Preso, ad es., l'ettaedro

$$
c \cdot a b d \cdot n m p
$$

si trova che, dei 35 triedri che esso contiene, 17 sono di $2^{\mathrm{a}}$ specie e 18 di $1^{\mathrm{a}}$. I 17 triedri di 2 specie sono: i quattro triedri del tetraedro $c a b d$, i quattro triedri del tetraedro $c n m p$, i triedri $c a n, c b m, c d p$, i triedri $a m p, b n p$, $d n m$, mad, nbd, pab.

Si ha adunque (posto $z=c, x_{1}=a, y_{1}=n, x_{2}=b, y_{2}=m, x_{3}=d, y_{3}=p$ ) la seguente proprietà: - I sette piani di un ettuedro principale si distribuiscono (in un modo solo) in due terne $x_{1} x_{2} x_{3}, y_{1} y_{2} y_{3}$ e in un piano $z$. Le due terne sono due triedri di $2^{a}$ specie. Il piano $z$ dà crigine a triedri di $2^{a}$ specie. con due piani qualunque di ciascuna terna e con ciascun piano $x_{i}$ dell'una accompagnato ad un piano $y_{i}$, corrispondente, dell'altra. Altri triedri di $2^{a}$ specie sono dati da un piano di una terna coi due non corrispondenti dell'altra $\left(x_{1} y_{2} y_{3}, x_{2} y_{1} y_{3}, \ldots x_{2} x_{3} y_{1}, \ldots\right)$. Si hanno cos̀̀ $17(=2+2 \cdot 3+3+2 \cdot 3)$ triedri di $2^{a}$ specie. I rimanenti $18(=3 \cdot 2+2 \cdot 3 \cdot 2)$ triedri $\left(z x_{1} y_{2}, z x_{1} y_{3}, \ldots\right.$ : $\left.x_{1} y_{1} y_{2}, x_{1} y_{1} y_{3}, \ldots y_{1} x_{1} x_{3}, \ldots\right)$ sono di $1^{a}$ specie.

Un ettaedro principale può scindersi in due modi e in due soltanto $\left(z x_{1} x_{2} x_{3}\right.$. $\left.y_{1} y_{2} y_{3} ; z y_{1} y_{2} y_{3} \cdot x_{1} x_{2} x_{3}\right)$ in un triedro di $2^{2}$ specie e in un tetraedro di cui i quattro triedri sono pure di $2^{a}$ specie. Laonde (n. ${ }^{\circ}$ 25) un ettcedro principale corrisponde a 2 pentaedri principali ed ogni pentaedro principale a 40 ettaedri principali: un ettaedro nascendo da un corrispondente pentaedro col sopprimerne una faccia e sostituirla con tre altre, che partano rispettivamente dalle tre rette della faccia medesima.

Ogni poliedro principale che contiene un tetraedro, di cui i quattro triedri sono di $2^{\mathrm{a}}$ specie, è necessariamente un ettaedro. Questa proprietà segue immediatamente dal $\mathrm{n} .^{\circ} 25$.

Un ettaedro principale può scomporsi in tre modi e in tre soltanto $\left(x_{1} x_{2} y_{1} y_{2}\right.$. $\left.z x_{3} y_{3}: x_{1} x_{3} y_{1} y_{3} \cdot z x_{2} y_{2} ; x_{2} x_{3} y_{2} y_{3} \cdot z x_{1} y_{1}\right)$ in un trielro di $2^{\mathrm{a}}$ specie $\mathrm{e}$ in un tetraedro di cui i quattro triedri sono di $1^{\mathrm{a}}$ specie $(*)$.

Siccome in ogni ettaedro principale esistono due tetraedri, i cui quattro triedri sono di $2^{a}$ specie e ogni tale tetraedro dà origine (n. $\left.{ }^{\circ} 25\right)$ a ctto cttaedri principali, il numero totale di questi è (n. $\left.{ }^{\circ} 24\right) \frac{8 \cdot 1080}{2}=4320$.

27. Prendasi di nuovo l'ettaedro del $n .^{\circ} 26$

$$
c \cdot a b d \cdot n m p \text { : }
$$

(") Non viceversa ogni ettaedro che contiene un cosifatto tatraedro è principale (cfr. $n .{ }^{\circ} 32$ ). 
essendo

$$
\begin{aligned}
& c \equiv c_{16} c_{23} c_{45}, \\
& a \equiv c_{14} c_{26} c_{35}, \\
& b \equiv c_{15} c_{24} c_{36}, \\
& d \equiv c_{46} c_{13} c_{25}, \\
& n \equiv a_{5} b_{6} c_{6}, \\
& m \equiv a_{1} b_{2} c_{12} \\
& p \equiv a_{3} b_{4} c_{34} .
\end{aligned}
$$

Levando dalle 27 rette le 21 in esso contenute, le sei rette residue $a_{2}, a_{4}, a_{6}$; $b_{1}, b_{3}, b_{5}$ giacciono sopra un iperboloide, ossia formano due terne complementari [ergänzende Tripel $(*)$ ].

Reciprocamente, si abbiano le due terne complementari $a_{2}, a_{4}, a_{6} ; b_{1}, b_{3}, b_{5}$ (che si possono ritenere affatto arbitrarie, ripetendo una considerazione fatta nel n. ${ }^{\circ} 25$ ). Le 21 rette rimanenti si distribuiscono (in un modo solo) in una bissestupla

$$
\begin{aligned}
& a_{1} a_{3} a_{5} c_{46} c_{62} c_{24} \\
& \begin{array}{lllll}
c_{35} c_{51} c_{13} b_{2} & b_{4} & b_{6}
\end{array}
\end{aligned}
$$

e in una coppia di triedri conjugati (di $3^{\mathrm{a}}$ specie)

$$
\left|\begin{array}{lll}
c_{16} & c_{23} & c_{45} \\
c_{34} & c_{56} & c_{12} \\
c_{25} & c_{14} & c_{36}
\end{array}\right| .
$$

Inoltre, esclusi i piani che contengono alcuna (una 0 due) delle sei rette $a_{2}$, $a_{4}, a_{6}, b_{1}, b_{3}, b_{5}$, rimangono 24 piani che si distribuiscono pure in due gruppi; di 6 , che sono quelli della coppia $(C)$, e di 18 , che passano a due a due per le rette di questa coppia e a tre a tre per le rette della bissestupla $(B)$. Un piano $c_{16} c_{23} c_{4}$, della coppia $(C)$ è piano $z\left(n^{\circ}{ }^{\circ} 26\right)$ di due ettaedri principali, che sono completati dai 12 piani del secondo gruppo, che passano per le rette di $(C)$ esterne al piano considerato; cioè dell' ettaedro suindicato $c \cdot a b d \cdot n m p$

\footnotetext{
(") Sturm, Ueber die 27 Geraden der cubischen Fläche (Math. Ann., tom. XXIII), pag. 290.
} 
e dell' ettaedro $\gamma \cdot \alpha \beta \delta \cdot \nu \mu \pi$, essendo

$$
\begin{aligned}
& \gamma \equiv c_{16} c_{23} c_{45}, \\
& \alpha \equiv c_{12} c_{35} c_{46}, \\
& \beta \equiv c_{56} c_{13} c_{42}, \\
& \delta \equiv c_{34} c_{26} c_{15}, \\
& \nu \equiv a_{3} b_{8} c_{86}, \\
& \mu \equiv a_{1} b_{4} c_{14}, \\
& \pi \equiv a_{5} b_{2} c_{52} .
\end{aligned}
$$

Adunque, escluse le sei rette di due terne complementari, nel sistema rimanente di 21 relte (e 24 piani) esistono 12 ettaedri principali, cioè quelle 21 rette si possono pensare, in 12 modi diversi, come l'intersezione completa della superficie di $3^{\circ}$ ordine e di 7 piani. I 12 ettaedri si dividono in sei paja: due ettaedri di un pajo hanno lo stesso piano z: e $i$ sei piani $z$ sono quelli di una coppia di triedri conjugati (*). Inoltre è evidente che ciascuno dei 12 ettaedri d̀̀ $i 24$ piani del sistema nei proprii 7 piani e nei 17 conjugati ai 17 triedri di $2^{a}$ specie in esso contenuti: la qual proprietà ha luogo altresì per gli enneaedri e per il pentaedro principale (n. ${ }^{\mathrm{i}} 18,23$ ).

Quindi $i 4320$ ettaedri principali si distribuiscono in 360 gruppi corrispondenti alle 360 coppie di terne complementari.

28. Discutiamo infine il $3^{\circ}$ caso ( $\mathrm{n}^{\circ} 22$ ), nel quale non si aggiunge ad $a b c$ alcuno dei piani $d, e$. Conservate le notazioni precedenti (n. $\left.{ }^{\circ} 25\right)$, supponiamo dapprima che si prendano tre dei piani $m, m^{\prime}, n, n^{\prime}, p, p^{\prime}$. Otto casi sono possibili, cioè si hanno otto poliedri principali

$$
\begin{array}{ll}
a b c m n p h, & a b c m^{\prime} n^{\prime} p^{\prime} h^{\prime}, \\
a b c m^{\prime} n p g, & a b c m n^{\prime} p^{\prime} g^{\prime}, \\
a b c m n^{\prime} p f, & a b c m^{\prime} n p^{\prime} f^{\prime}, \\
a b c m n p^{\prime} h f^{\prime} g^{\prime}, & a b c m^{\prime} n^{\prime} p h^{\prime} f g .
\end{array}
$$

Dei quali i primi sei sono degli ettaedri principali già considerati (n. ${ }^{\circ} 26$ ), perchè contengono rispettivamente i tetraedri $c n m p, c n^{\prime} m^{\prime} p^{\prime}, b n m^{\prime} p, b n^{\prime} m p^{\prime}$,

(*) Altre proprietá del sistema di 21 rette, qui considerato, per ciò che riguarda la configurazione delle rette e particolarmente gli esagoni e doppî-esagoni (Sechsseit, Donpelsechsseit) che si possono formare colle rette stesse, trovansi nel $\$ 3$ del citato lavoro di Sturm (Math. Ann., tom. XXIII). 
$a n^{\prime} m p$, $a n m^{\prime} p^{\prime}$, in ognuno dei quali entrano quattro triedri di $2^{\bar{a}}$ specie, come risulta dall' osservare gli otto ettaedri del n. ${ }^{\circ} 25$ : e gli ultimi due poliedri cono enneaedri [di $2^{a}$ specie, perchè contengono triedri $a b c, \ldots$ di $2^{a}$ specie (cfr. n. $\left.\left.{ }^{\circ} 18\right)\right]$.

Le stesse considerazioni valgono, prendendo tre dei sei piani $f, f^{\prime}, g, g^{\prime}, h, h^{\prime}$.

Quindi rimangono a studiare i casi in cui si escludano due dei piani $f, f^{\prime}$, $g, g^{\prime}, h, h^{\prime}$, aventi una retta comune (cioè $f, f^{\prime}, \circ g, g^{\prime}, 0 h, h^{\prime}$ ) e insieme due dei piani $m, m^{\prime}, n, n^{\prime}, p, p^{\prime}$ che abbiano pure una retta comune (ciò $m, m^{\prime}$, o $\left.n, n^{\prime}, 0 p, p^{\prime}\right)$.

$\mathrm{Ma}$, per escludere i piani $f, f^{\prime}$, occorre aggiungere una delle due coppie $n p$, $n^{\prime} p^{\prime}$ e per conseguenza escludere $\mathrm{i}$ piani $m, m^{\prime}$. Parimenti, escludendo $g, g^{\prime}$, deve escludersi $n, n^{\prime}$ ed escludendo $h, h^{\prime}$, deve escludersi $p, p^{\prime}$. Però i tre casi che così si presentano non sono sostanzialmente differenti, dal primo caso nascendo il secondo e dal secondo il terzo col fare ordinatamente gli scambî

$$
\begin{aligned}
& 3,4 ; \quad 1,6 ; 2,5 \text { : } \\
& 1,2 ; 3,5 ; \quad 4,6 \text {. }
\end{aligned}
$$

Onde basta considerare il primo caso, nel quale si hanno i due poliedri principali

$$
a b c g h n p, \quad a b c g^{\prime} h^{\prime} n^{\prime} p^{\prime} \text {. }
$$

Ora, come si verifica facilmente, i quattro triedri di ciascuno dei tetraedri $a b h p, a c g n, a b h^{\prime} p^{\prime}, a c g^{\prime} n^{\prime}$ sono di $2^{a}$ specie e per conseguenza (n. ${ }^{\circ} 25$ ) $i$ due suddetti poliedri sono degli ettaedri principali studiati precedentemente (n. $\left.{ }^{\circ} 26\right)$.

Si conclude che nel $3^{\circ}$ caso ( $n .^{\circ}$ 22) non si ha alcun nuovo poliedro principale.

\section{$\S 9$. Poliedri che contengono almeno un triedro di $1^{a}$ specie.}

29. E, come adesso mostreremo, non si ha alcun nuovo poliedro principale neppure nel caso $B$ ) del $n .^{\circ} 21$ : ossia $i$ poliedri principali che contengono almeno un triedro di $1^{a}$ specie sono di quelli già considerati (ciò̀ enneaedri o pentaedri principali o ettaedri principali).

Sia $a b c$ un triedro di $1^{\text {a }}$ specie e $a^{\prime} b^{\prime} c^{\prime}, a^{\prime \prime} b^{\prime \prime} c^{\prime \prime}$ gli altri due triedri di $1^{\text {a }}$ specie che con esso formano un ciclo (n. ${ }^{\circ} 20$ ). Per costruire poliedri contenenti $a b c$, dobbiamo escludere, oltre $a, b, c, \mathrm{i}$ nove piani dei triedri conjugati ai triedri 
(di 3 specie) $a b c^{\prime}, a b^{\prime} c, a^{\prime} b c$ e i rimanenti piani che passano per le rette di $a, b, c$ (due piani per ciascuna retta): cosicchè restano

$$
45-3-9-3 \cdot 6=15
$$

piani. Questi piani sono $a^{\prime}, b^{\prime}, c^{\prime}, a^{\prime \prime}, b^{\prime \prime}, c^{\prime \prime}$ e $\mathrm{i}$ nove piani dei triedri conjugati ai triedri (di $3^{\mathrm{a}}$ specie) $a^{\prime} b^{\prime} c^{\prime \prime}, b^{\prime} c^{\prime} a^{\prime \prime}, c^{\prime} a^{\prime} b^{\prime \prime}$, giacchè tali piani contengono le rette di $a^{\prime}, b^{\prime}, c^{\prime \prime}, b^{\prime} \ldots$ e però non quelle di $a, b, c$. Indichiamo $\mathrm{i}$ detti nove piani con $\alpha, \beta, \gamma, \alpha^{\prime}, \beta^{\prime}, \gamma^{\prime}, \alpha^{\prime \prime}, \beta^{\prime \prime}, \gamma^{\prime \prime}$, e precisamente

$$
\begin{gathered}
\text { con } \alpha \beta \gamma \text { il triedro conjugato ad } a^{\prime} b^{\prime} c^{\prime \prime}, \\
" \alpha^{\prime} \beta^{\prime} \gamma^{\prime} ", \quad b^{\prime} c^{\prime} a^{\prime \prime}, \\
" \alpha^{\prime \prime} \beta^{\prime \prime} \gamma^{\prime \prime} "
\end{gathered}
$$

Anzitutto si avverta che esistono i quattro enneaedri

$$
\begin{aligned}
& a b c a^{\prime} b^{\prime} c^{\prime} a^{\prime \prime} b^{\prime \prime} c^{\prime \prime}, \\
& a b c^{\prime} \alpha \beta \gamma a^{\prime \prime} b^{\prime \prime} c, \\
& b c a^{\prime} \alpha^{\prime} \beta^{\prime} \gamma^{\prime} b^{\prime \prime} c^{\prime \prime} a, \\
& c a b^{\prime} \alpha^{\prime \prime} \beta^{\prime \prime} \gamma^{\prime \prime} c^{\prime \prime} a^{\prime \prime} b ;
\end{aligned}
$$

il primo dei quali è di $1^{\mathrm{a}}$ specie (n. $\left.{ }^{\circ} 20\right)$ e gli altri tre sono di $2^{\mathrm{a}}$ specie (n. ${ }^{\circ} 13$ ).

Poi si osservi che le tre rette di $a^{\prime}$ appartengono ordinatamente ad $\alpha, \beta, \gamma$ e parimenti ad $\alpha^{\prime \prime}, \beta^{\prime \prime}, \gamma^{\prime \prime}$. Onde questi tre piani avranno con quei tre rispettivamente una retta comune. Potendosi scambiare i simboli $\alpha, \beta, \gamma$ e cosi $\alpha^{\prime}, \beta^{\prime}, \gamma^{\prime}$, come pure $\alpha^{\prime \prime}, \beta^{\prime \prime}, \gamma^{\prime \prime}$, si può fissare che le tre rette di $\alpha^{\prime}$ sieno

$$
\alpha \alpha^{\prime \prime}, \quad \beta \beta^{\prime \prime}, \quad \gamma \gamma^{\prime \prime} \text { : }
$$

e similmente che le tre rette di $c^{\prime}$ sieno

$$
\alpha^{\prime} \alpha^{\prime \prime}, \quad \beta^{\prime} \xi^{\prime \prime}, \quad \gamma^{\prime} \gamma^{\prime \prime} \text {. }
$$

Le tre rette di $b^{\prime}$ debbono essere ordinatamente di intersezione dei piani $\alpha, \beta, \gamma$ coi piani $\alpha^{\prime}, \beta^{\prime}, \gamma^{\prime}$. Ma non può a incontrare $\alpha^{\prime}$ in una retta della superficie, poichè, per la notazione ora posta, $\alpha, \alpha^{\prime}$ hanno ciascuno con $\alpha^{\prime \prime}$ una tale retta comune, e queste due rette, appartenendo ad $a^{\prime}, c^{\prime}$, sono distinte. Si può stabilire che $\alpha$ incontri $\beta^{\prime}$ in una retta della superficie; perchè, se incontrasse invece $\gamma^{\prime}$, basterehbe, come è permesso, fare gli scambi $\beta, \gamma ; \beta^{\prime}, \gamma^{\prime} ; \beta^{\prime \prime}, \gamma^{\prime \prime}$. Ma allora il piano $\beta$, che non può avere retta della superficie a comune con $\beta^{\prime}$, per la ragione di dianzi, nemmeno può averla con $\alpha^{\prime}$, giacchè, se ciò acca- 
desse, i residui piani $\gamma, \gamma^{\prime}$ dovrebbero incontrarsi in una retta della superficie, ciò che, per la stessa ragione, non può essere. Dunque le tre rette di $b^{\prime}$ sono

$$
\text { a. } \beta^{\prime}, \quad \beta \gamma^{\prime}, \quad \gamma \alpha^{\prime} \text {. }
$$

Raccogliendo, si hanno quindi le seguenti rette della superficie (essendo $a \alpha . \alpha^{\prime \prime}$ retta comune ai piani $a, \alpha, \alpha^{\prime \prime}$; ecc.):

$$
\begin{array}{rrr}
a^{\prime} \alpha \alpha^{\prime \prime}, & a^{\prime} \beta \beta^{\prime}, & a^{\prime} \gamma \gamma^{\prime \prime}, \\
c^{\prime} \alpha^{\prime} \alpha^{\prime \prime}, & c^{\prime} \beta^{\prime} \beta^{\prime \prime}, & c^{\prime} \gamma^{\prime} \gamma^{\prime \prime}, \\
b^{\prime} \alpha \beta^{\prime}, & b^{\prime} \beta \gamma^{\prime}, & b^{\prime} \gamma \alpha^{\prime}, \\
c^{\prime \prime} \alpha, & c^{\prime \prime} \beta, & c^{\prime \prime} \gamma, \\
a^{\prime \prime} \alpha^{\prime}, & a^{\prime \prime} \beta^{\prime}, & a^{\prime} \gamma^{\prime}, \\
b^{\prime \prime} \alpha^{\prime \prime}, & b^{\prime \prime} \beta^{\prime \prime}, & b^{\prime} \gamma^{\prime \prime} .
\end{array}
$$

Distinguiamo i poliedri contenenti $a b c$, ciò̀ che si ottengono coll' aggiungere ad $a b c$ piani scelti fra i quindici $a^{\prime}, b^{\prime}, c^{\prime}, a^{\prime \prime}, b^{\prime \prime}, c^{\prime \prime}, \alpha_{i} \beta, \gamma, a^{\prime}, \beta^{\prime}, \gamma^{\prime}, \alpha^{\prime \prime}, \beta^{\prime \prime}, \gamma^{\prime \prime}$, in tre classi:

1. ${ }^{a}$ Classe. - Aggiungasi ad a $b c$ almeno due dei piani $a^{\prime}, b^{\prime}, c^{\prime}, a^{\prime \prime}, b^{\prime \prime}, c^{\prime \prime}$. Si hanno i seguenti quattro casi (poichè $a b c a^{\prime} c^{\prime} \ldots$ si tratterebbe come $a b c a^{\prime} b^{\prime} \ldots ;$ ecc.):

$$
\begin{aligned}
& a b c a^{\prime} b^{\prime} \ldots, \\
& a b c a^{\prime} a^{\prime \prime} \ldots, \\
& a b c a^{\prime \prime} b^{\prime \prime} \ldots, \\
& a b c a^{\prime} b^{\prime \prime} \ldots .
\end{aligned}
$$

Nel primo e secondo, esistendo le rette $(H)$, non si può aggiungere alcuno dei piani $\alpha, \beta, \gamma, \alpha^{\prime}, \beta^{\prime}, \gamma^{\prime}, \alpha^{\prime \prime}, \beta^{\prime \prime}, \gamma^{\prime \prime}$ e però si hanno poliedri contenuti nell'enneaedro $\left(E_{1}\right)$. Nel terzo caso sono disponibili $a^{\prime}, b^{\prime}, c^{\prime}, c^{\prime \prime}, \alpha, \beta, \gamma$ : ma devonsi escludere $a^{\prime}, l^{\prime}$ che dànno nuovamente il secondo caso $\left(a b c a^{\prime} a^{\prime \prime} \ldots ; a b c b^{\prime} b^{\prime \prime} \ldots\right)$. Dei piani rimanenti se si aggiunge $c^{\prime}$, non si aggiungerà $c^{\prime \prime}$ per non ricadere nello stesso secondo caso, ma si potranno aggiungere $\alpha, \beta, \gamma$ e si otterrà un poliedro contenuto nell' enneaedro $\left(E_{2}^{\prime}\right)$. Che se escludasi $c^{\prime}$, potrà aggiungersi $c^{\prime \prime}$ e allora non $\alpha, \beta, \gamma$; ovvero potranno aggiungersi $\alpha, \beta, \gamma$ e allora non $c^{\prime \prime}$ : cioè si otterrà un poliedro contenuto in $\left(E_{1}\right) \circ\left(E_{2}\right)$. Finalmente nel quarto caso ponno aggiungersi $b^{\prime}, c^{\prime}, a^{\prime \prime}, c^{\prime \prime}, a^{\prime}, \beta^{\prime}, \gamma^{\prime}$ : ma devono escludersi $b^{\prime}, c^{\prime}, a^{\prime}, c^{\prime \prime}$, 
perchè si ritorna al primo, secondo e terzo caso già esaminati; e, aggiungendo $\alpha^{\prime}, \beta^{\prime}, \gamma^{\prime}$, si ottiene un poliedro contenuto in $\left(E_{2}^{\prime}\right)$.

$2 .^{a}$ Classe. - Aggiungasi ad $a b c$ uno solo dei piani $a^{\prime}, b^{\prime}, c^{\prime}, a^{\prime \prime}, b^{\prime \prime}, c^{\prime \prime}$. Si hanno due casi essenzialmente distinti:

$$
\begin{aligned}
& a b c a^{\prime} \ldots \\
& a b c a^{\prime \prime} \ldots
\end{aligned}
$$

ove s'intende che $\mathrm{i}$ piani da aggiungere sono dei nove $\alpha, \beta, \gamma, \alpha^{\prime}, \beta^{\prime}, \gamma^{\prime}, \alpha^{\prime \prime}$, $\beta^{\prime \prime}, \gamma^{\prime \prime}$. Ma, per le $(H)$, nel primo caso ponno aggiungersi soltanto $\alpha^{\prime}, \beta^{\prime}, \gamma^{\prime}$ e nel secondo soltanto $\alpha, \beta, \gamma, \alpha^{\prime \prime}, \beta^{\prime \prime}, \gamma^{\prime \prime}$ : e si ottengono poliedri già discussi (n. $\left.{ }^{\circ} 22\right)$, perchè contenenti triedri di $2^{a}$ specie. Cioè sono di $z^{a}$ specie (nel primo caso),

$$
\begin{array}{lll}
a a^{\prime} \alpha^{\prime}, & a \alpha^{\prime} \beta, & a \gamma^{\prime}, \\
a b \alpha^{\prime}, & a b \beta^{\prime}, & a b \gamma^{\prime}, \\
a c \alpha^{\prime}, & a c \beta^{\prime}, & a c \gamma^{\prime},
\end{array}
$$

\begin{tabular}{|c|c|c|}
\hline$a a^{\prime \prime} \propto$ & $a a^{\prime \prime} \beta$, & $a a^{\prime \prime} \cdot \gamma$, \\
\hline$a a^{\prime \prime} \alpha^{\prime \prime}$, & $a a^{\prime \prime} \beta^{\prime \prime}$ & $a a^{\prime \prime} \gamma^{\prime \prime}$, \\
\hline$a c \alpha$, & $a c \beta$ & $a c \gamma$, \\
\hline$b c \alpha$, & $b c \beta$ & $b c \gamma$, \\
\hline$a b \quad \alpha^{\prime \prime}$, & $a b \quad \beta^{\prime \prime}$ & $a b \gamma^{\prime \prime}$, \\
\hline$b c \quad a^{\prime \prime}$, & $b c \quad \beta^{\prime \prime}$, & $b c$ \\
\hline
\end{tabular}

e (nel secondo caso),

I precedenti triedri provengono infatti dagli enneaedri di $2^{\text {a }}$ specie $\left(E_{2}\right),\left(E_{2}^{\prime}\right)$, $\left(E_{2}^{\prime \prime}\right)$ prendendo in ogni enneaedro tre piani appartenenti rispettivamente ai tre triedri di $3^{\prime}$ specie di esso (n. $\left.{ }^{\circ} 18\right)$.

$3^{a}$ Classe. - Aggiungasi ad abc nessuno dei piani $a^{\prime}, b^{\prime}, c^{\prime}, a^{\prime \prime}, b^{\prime \prime}, c^{\prime}$ e però soltanto dei piani $\alpha, \beta, \gamma, \alpha^{\prime}, \beta^{\prime}, \gamma^{\prime}, \alpha^{\prime \prime}, \beta^{\prime \prime}, \gamma^{\prime \prime}$. Per l' esistenza dei triedri di $2^{2}$ specie ora indicati, anche qui si hanno poliedri già studiati.

30. Si ha adunque il teorema:

I poliedri principali sono l' enneaedro di $1^{a}$ specie, l'enneaedro di $2^{a}$ specie, l'ettaedro e il pentaedro (principali) considerati nei $\$ \$ 7$, 8. Cioè: qualunque poliedro che si può formare coi 45 piani tritangenti $\grave{e}$ uno dei quattro precedenti o è contenuto in essi. 


\section{$\S 10$. Tetraedri.}

31. Ci proponiamo ora, coll'ajuto del precedente teorema, di procedere alla determinazione di tutti i possibili poliedri. A tal fine introdurremo la notazione $\left(t_{1}, t_{2}, t_{3}\right)^{n}$ per indicare un poliedro di ordine $n, \mathrm{i}$ cui triedri sono $t_{1} \mathrm{di}$ $1^{\mathrm{a}}$ specie, $t_{2}$ di $2^{\mathrm{a}}$ e $t_{3}$ di $3^{\mathrm{a}}$ (cfr. n. $^{\circ} 40$ ): onde sarà

$$
t_{1}+t_{2}+t_{3}=\frac{u(n-1)(n-2)}{1 \cdot 2 \cdot 3} \text {. }
$$

$\mathrm{E}$, cominciando dai tetraedri, consideriamo dapprima quelli nascenti da un enneaedro di $1^{\text {a }}$ specie

$$
a_{1} a_{2} a_{3} a_{4} a_{5} a_{6} a_{7} a_{8} a_{9}
$$

di cui $\mathrm{i}$ triedri di $3^{\text {n }}$ specie sieno dati dal determinante $\left(\mathrm{n}^{\circ} 4\right)$

$$
\begin{array}{lll|l}
a_{1} & a_{2} & a_{3} & \\
a_{4} & a_{5} & a_{6} & \left.{ }^{*}\right) . \\
a_{7} & a_{8} & a_{9} &
\end{array}
$$

Si hanno due specie essenzialmente distinte, $(4,0,0)^{4},(3,0,1)^{4}$. Poichè se si prenda un triedro di $1^{\mathrm{a}}$ specie $a_{1} a_{4} a_{8}$ e si considerino $\mathrm{i}$ due altri triedri di $1^{\mathrm{a}}$ specie $a_{3} a_{6} a_{7}, a_{2} a_{5} a_{5}$ che con quello formano un ciclo, è evidente (n. ${ }^{\circ} 20$ ) che $\mathrm{i}$ tre tetraedri $a_{1} a_{4} a_{8} a_{2}, a_{1} a_{4} a_{8} a_{5}, a_{1} a_{4} a_{8} a_{9}$ contengono ciascuno quattro triedri di $1^{a}$ specie e sono $\mathrm{i}$ soli esistenti nell'enneaedro e aventi a comune il triedro $a_{1} a_{4} a_{8}$, i quali godono di tale proprietà. Dall' enneaedro nascono quindi (n..$^{\circ}$ 18) $54=\frac{72 \cdot 3}{4}$ tetraedri $(4,0,0)^{4}$ e però, non potendo due enneaedri di $1^{a}$ specie avere a comune un tetraedro (cfr. $n .^{\circ} 14$ ), il numero totale di quei tetraedri è $2160=54 \cdot 40$. Che se un tetraedro $a_{1} a_{2} a_{3} a_{i}$, proveniente dall' enneaedro, contiene un triedro di $3^{\text {a }}$ specie $a_{1} a_{2} a_{3}$, gli altri tre triedri sono di $1^{3}$ specie $\left(n .^{0} 18\right)$, cioè si ha un tetraedro $(3,0,1)^{4}$. Di questi, in ogni enne.

() A questo determinante, per la determinazione dei sunnominati triedri, si possono sostituire altri cingne, por es.,

eec.

$$
\left|\begin{array}{lll}
a_{1} & a_{2} & a_{3} \\
a_{5} & a_{6} & a_{4} \\
a_{9} & a_{7} & a_{8}
\end{array}\right|
$$


aedro, esistono $72=12 \cdot 6$ e però il loro numero totale è $2880=72 \cdot 40$ : il che segue altresì dal notare che un triedro di $3^{a}$ specie è comune a due enneaedri di $1^{a}$ specie $\left(n^{0} 7\right)$ e quindi entra in 12 tetraedri $(3,0,1)^{4}$.

Sia ora

$$
b_{1} b_{2} b_{3} \cdot b_{4} b_{5} b_{6} \cdot b_{7} b_{8} b_{9}
$$

un enneaedro di $2^{a}$ specie, le tre terne in cui è diviso essendo i suoi tre triedri di $3^{\mathrm{a}}$ specie. Si hanno tre specie di tetraedri dei tipi

$$
\begin{aligned}
& b_{1} b_{2} b_{3} b_{4}, \\
& b_{1} b_{2} b_{4} b_{5}, \\
& b_{1} b_{2} b_{4} b_{7} .
\end{aligned}
$$

Il primo e il secondo (n..$\left.^{0} 18\right)$ sono rispettivamente tetraedri $(3,0,1)^{4},(4,0,0)^{4}$. Il terzo è un tetraedro $(2,2,0)$. Il numero di questi ultimi contenuti nell'enneaedro è manifestamente $81=3 \cdot 9 \cdot 3$ e per conseguenza il loro numero totale è $12960=81 \cdot 160$; poichè due enneaedri di $2^{\mathrm{a}}$ specie possono avere soltanto a comune un tetraedro $(4,0,0)^{4}$ (cfr. Nota al n..$\left.^{\circ}\right\rfloor$ ).

Infine dall' ettaedro principale $\left(\mathrm{n}^{\mathrm{0}}{ }^{26}\right)$

$$
z \cdot x_{1} x_{2} x_{3} \cdot y_{1} y_{2} y_{3}
$$

si hanno tetraedri di questi tipi

$$
\begin{array}{ll}
z x_{1} x_{2} x_{3}, & x_{1} x_{2} x_{3} y_{1}, \\
z x_{1} x_{2} y_{1}, & x_{1} x_{2} y_{1} y_{3}, \\
z x_{1} x_{2} y_{3}, & x_{1} x_{2} y_{1} y_{3} ;
\end{array}
$$

dej quali il primo a sinistra ̀̀ un tetraedro $(0,4,0)^{4}$, di quelli (i soli) pure contenuti in un pentaedro principale e dei quali già trovammo il numero totale (n. $\left.{ }^{0} 24\right)$; l' nltimo a destra è un tetraedro $(4,0,0)^{4}$ e i rimanenti sono tetraedri $(2,2,0)^{4}$.

Che i tetraedri $(4,0,0)^{4}$ contenuti nell'enneaedro di $1^{4}$ specie siano di quelli esistenti in un enneaedro di $2^{a}$ specie e in un ettaedro principale e che la stessa cosa si verifichi per i tetraedri $(3,0,1)^{4}$ che si trovano nelle due specie di enneaedri e per i tetraedri $(2,2,0)^{4}$ che stanno nell'enneaedro di $2^{a}$ specie e nell'ettaedro principale, risulta dal teorema seguente.

32. Un tetraedro di cui $i$ quattro triedri sono di $1^{a}$ specie, ovvero tre di $1^{*}$ specie e uno di $3^{a}$, giace in un ennededro di $1^{a}$ specie e in uno di $2^{a}$ specie. Un tetraedro di cui due triedri sono di $1^{a}$ specie e due di $2^{a}$, giace in un en- 
neaedro di $2^{a}$ specie. Giacchè, se $a b c$ è un triedro di $1^{\text {a }}$ specie e si riprendono le considerazioni e le notazioni $\operatorname{del} \S 9$, si possono aggiungere ad $a b c$, quindici soli piani $a^{\prime}, b^{\prime}, c^{\prime}, a^{\prime \prime}, b^{\prime \prime}, c^{\prime \prime}, \alpha, \beta, \gamma, \alpha^{\prime}, \beta^{\prime}, \gamma^{\prime}, \alpha^{\prime \prime}, \beta^{\prime \prime}, \gamma^{\prime \prime}$; onde si ottengono 15 tetraedri a cui appartiene $a b c$. Dei quali, i tetraedri

$$
a b c a^{\prime \prime}, \quad a b c b^{\prime \prime}, \quad a b c c^{\prime \prime},
$$

contenenti ciascuno quattro triedri di $1^{i l}$ specie (n..$\left.^{\circ} 18\right)$, esistono nell'enneaedro di $1^{2}$ specie $\left(E_{1}\right)$ e rispettivamente negli enneaedri di $2^{2}$ specic $\left(E_{2}\right),\left(E_{2}^{\prime}\right)$, $\left(E^{\prime \prime}\right)$ : i tetraedri

$$
a b c c^{\prime}, \quad a b c a^{\prime}, \quad a b c b^{\prime},
$$

aventi ognuno tre triedri di $1^{\text {a }}$ specie e uno di $3^{a}$, stanno pure in $\left(E_{1}\right)$ e ordinatamente in $\left(E_{2}\right),\left(E_{2}^{\prime}\right),\left(E_{2}^{\prime \prime}\right)$ : e infine $\mathrm{j}$ tetraedri

$$
\begin{array}{lll}
a b c \alpha, & a b c \beta, & a b c \gamma, \\
a b c \alpha^{\prime}, & a b c \beta^{\prime}, & a b c \gamma^{\prime}, \\
a b c \alpha^{\prime \prime}, & a b c \beta^{\prime \prime}, & a b c \gamma^{\prime \prime},
\end{array}
$$

cadauno con due triedri di $1^{\mathrm{a}}$ specie e due di 2 , giacciono rispettivamente nei detti enneaedri $\left(E_{2}\right),\left(E_{2}^{\prime}\right),\left(E_{2}^{\prime \prime}\right)$ : c. d. d.

\section{$\S$ 11. Pentaedri.}

33. Per ottenere i poliedri di ordine $n>4$ contenuti in un enneaedro di $1^{:}$specie, è utile osservare che esister $\grave{a}$ in essi almeno un triedro di $3^{\alpha}$ specie. Poichè, considerando uno dei cicli di triedri di 3 specie $a_{1} a_{4} a_{8}, a_{3} a_{6} u_{7}, a_{2} a_{5} a_{9}$ contenuti nell'enneaedro (.$^{\circ} 20$ ), se non si aggiunge ad $a_{1} a_{4} a_{8}$ alcuno dei piani $a_{3}, a_{6}, a_{7}$, dovranno aggiungersi almeno due dei piani $a_{2}, a_{5}, a_{9}$, e però ecc.

Quindi, mantenendo per un enneaedro di $1^{\text {a }}$ specie le notazioni $\left(E_{1}\right),(D)$ del $\S 10$, si hanno $i$ due tipi di pentaedri

$$
\begin{aligned}
& a_{1} a_{2} a_{3} a_{4} a_{7} \\
& a_{1} a_{2} a_{3} a_{4} a_{5}
\end{aligned}
$$

(secondochè il quinto piano $a_{7}$ dà col quarto $a_{4}$ un triedro di $3^{2}$ specie completato dai piani $a_{1}, a_{2}, a_{3}$, oppur no), che sono rispettivamente $(8,0,2)^{\text {s, }}$, $(9,0,1)^{5}$. Il numero dei primi è $2160=(6 \cdot 9) \cdot 40$ e quello dei secondi è $2880=(6 \cdot 12) \cdot 40$. 
Da un enneaedro di $2^{\mathrm{a}}$ specie $\left(E_{2}\right)(\S 10)$, si ottengono questi tipi

$$
\begin{aligned}
& b_{1} b_{2} b_{3} b_{4} b_{5} \\
& b_{1} b_{2} b_{3} b_{4} b_{7} \\
& b_{1} b_{2} b_{4} b_{5} b_{7}
\end{aligned}
$$

il primo dei quali è $(9,0,1)^{5}$ ed è di quelli trovati dianzi, giacchè nasce anche dall' enneaedro di $1^{a}$ specie, che $\left(n .^{\circ} 13\right)$ proviene da $\left(E_{2}\right)$ col sostituire a $b_{7} b_{8} b_{9}$ il triedro conjugato. Il secondo e il terzo sono $(6,3,1)^{5},(6,4,0)^{5}$. Si hanno $4320=(3 \cdot 3 \cdot 3) \cdot 160$ poliedri $(6,3,1)^{5}$ e $12960=(3 \cdot 3 \cdot 3 \cdot 3) \cdot 160$ poliedri $(6,4,0)^{5}$.

Da un ettaedro $(P)$ derivano i tipi

$$
\begin{aligned}
& z x_{1} x_{2} x_{3} y_{1}, \\
& z x_{1} x_{2} y_{1} y_{2}, \\
& z x_{1} x_{2} y_{1} y_{3}, \\
& x_{1} x_{2} x_{3} y_{1} y_{2} .
\end{aligned}
$$

Il secondo e il quarto sono pentaedri $(6,4,0)^{5}$. Il primo è un pentaedro $(4,6,0)^{5}$ e il terzo $(5,5,0)^{5}$.

In un pentaedro $(4,6,0)^{5} \mathrm{c}^{\prime} \mathrm{è}\left(\mathrm{n} .^{\circ} 26\right)$ un tetraedro (e uno solo) $z x_{1} x_{2} x_{3}$, di cui ogni triedro è di $2^{a}$ specie. $\mathrm{E}$ da un cosifatto tetraedro nascono sei pentaedri di quella specie [cfr. n. ${ }^{\circ} 25\left(^{*}\right)$ ]. Adunque (n. $\left.{ }^{\circ} 24\right)$ il numero dei pentaedri $(4,6,0)^{5}$ è $6480=1080 \cdot 6$. Donde risulta che da un tetraedro $(2,2,0)^{4}$ si ricavano due (e due soli) pentaedri $(4,6,0)^{5}$, giacchè in un tal pentaedro, esistono quattro di quei tetraedri $\left(z x_{1} x_{2} y_{1}, z x_{1} x_{3} y_{1}, z x_{2} x_{3} y_{1}, x_{1} x_{2} x_{3} y_{1}\right)$ e si ha appunto (n. $\left.{ }^{\circ} 31\right) \frac{12960 \cdot 2}{4}=6480$. Determineremo fra poco (n..$\left.^{\circ} 35\right)$ il numero dei pentaedri $(5,5,0)^{5}$. Intanto qui osserviamo che $i$ cinque tetraedri contenuti in un tale pentaedro sono tutti $(2,2,0)^{4}$.

I pentaedri $(6,4,0)^{5}$ provenienti dall' ettaedro principale sono di quelli che provengono dall' enneaedro di $2^{a}$ specie, in virtù del teorema seguente.

() Prese le indicazioni di questo numero, i sei lentaedri sono

$$
\begin{array}{lll}
a b c d n, & a b c d m, & a b c d p, \\
a b c d n^{\prime}, & a b c d m^{\prime}, & a b c d p^{\prime} .
\end{array}
$$


34. Un pentaedro, $i$ cui 10 triedri sono 6 di $1^{a}$ specie e 4 di $2^{a}$ specie, esiste in un enneaedro di $2^{a}$ specie.

In vero i pentaedri, colla detta proprieta, contengono per il numero precedente, un tetraedro i cui quattro triedri sono di $1^{\text {a }}$ specie (cioè $b_{1} b_{2} b_{4} b_{5} b_{7}$ il tetraedro $b_{1} b_{2} b_{4} b_{5}$ e $z x_{1} x_{2} y_{1} y_{2}, x_{1} x_{2} x_{3} y_{1} y_{2}$, il tetraedro $x_{1} x_{2} y_{1} y_{2}$ ). Ora, ripigliando le notazioni del $\S 9$, i soli tetraedri a cui dà origine un triedro di $1^{a}$ specie $a b c$, aventi gli altri tre triedri pure di $1^{a}$ specie, sono (n. $\left.{ }^{\circ} 32\right)$

$$
a b c a^{\prime \prime}, \quad a b c b^{\prime \prime}, \quad a b c c^{\prime \prime} \text {. }
$$

Ma, esistendo l'enneaedro $\left(E_{1}\right)$, non si hanno (cfr. $n .^{0} 33$ ) pentaedri della specie voluta coll' aggiungere $a^{\prime}, b^{\prime}, c^{\prime}$ : mentre aggiungendo gli altri piani $\alpha, \beta, \gamma$, $\alpha^{\prime}, \beta^{\prime}, \gamma^{\prime}, \alpha^{\prime \prime}, \beta^{\prime \prime}, \gamma^{\prime \prime}$, si ottengono, tenendo presenti le rette $(H)$, i soli pentaedri

$$
\begin{array}{lll}
a b c a^{\prime \prime} \alpha, & a b c b^{\prime \prime} \alpha, & a b c c^{\prime \prime} \alpha^{\prime}, \\
a b c a^{\prime \prime} \beta, & a b c b^{\prime \prime} \beta, & a b c c^{\prime \prime} \beta^{\prime}, \\
a b c a^{\prime \prime} \gamma, & a b c b^{\prime \prime} \gamma, & a b c c^{\prime \prime} \gamma^{\prime}, \\
a b c a^{\prime \prime} \alpha^{\prime \prime}, & a b c b^{\prime \prime} \alpha^{\prime}, & a b c c^{\prime \prime} \alpha^{\prime \prime}, \\
a b c a^{\prime \prime} \beta^{\prime \prime}, & a b c b^{\prime \prime} \beta^{\prime}, & a b c c^{\prime \prime} \beta^{\prime \prime}, \\
a b c a^{\prime \prime} \gamma^{\prime \prime}, & a b c b^{\prime \prime} \gamma^{\prime}, & a b c c^{\prime \prime} \gamma^{\prime \prime}:
\end{array}
$$

i quali sono contenuti negli enneaedri di $2^{\text {a }}$ specie $\left(E_{2}\right),\left(E_{2}^{\prime}\right),\left(E_{2}^{\prime \prime}\right)$ : c. d. d.

35. E, continuando nelle stesse notazioni, si osservi che un tetraedro $(2,2,0)^{4}$, ad es. il tetraedro (n. $\left.{ }^{\circ} 32\right)$ a bca, appartiene a nove (soli) pentaedri

$$
\begin{gathered}
a b c \alpha \beta, \quad a b c \alpha \gamma, \quad a b c \alpha c^{\prime}, \quad a b c \alpha a^{\prime \prime}, \quad a b c \alpha b^{\prime \prime}, \\
a b c \alpha \alpha^{\prime}, \quad a b c \alpha \gamma^{\prime}, \quad a b c \alpha \beta^{\prime \prime}, \quad a b c \alpha \gamma^{\prime \prime}:
\end{gathered}
$$

come è chiaro, osservando le rette $(H)$. Di questi i primi cinque sono contenuti nell'enneaedro $\left(E_{\varepsilon}\right)$; mentre i quattro rimanenti non sono contenuti in alcun enneaedro. In vero il tetraedro $a b c \alpha$ appartiene al solo enneaedro di $2^{a}$ specie $\left(E_{*}\right)$ (Nota al n. $\left.{ }^{\circ} 15\right)$ e il triedro $a b c$ al solo enneaedro di $1^{\text {a }}$ specie $\left(E_{1}\right)$ (n. 19): nei quali due enneaedri non esistono i detti quattro pentaedri. Se ne trae (n. 33,34 ) che questi quattro pentaedri sono quelli contenuti soltanto nell'ettaedro principale, cioè $(4,6,0)^{5},(5,5,0)^{5}$ e precisamente due dei primi $\left(\mathrm{n} .{ }^{\circ} 33\right)$ e quindi due dei secondi. Adunque, ogni tetraedro $(2,2,0)^{4}$ ̀े co- 
mune a due pentaedri $(5,5,0)^{5}$ e però, in un tal pentaedro esistendo (n. 33 ) cinque tetraedri $(2,2,0)^{4}$, il numero dei pentaedri $(5,5,0)^{5}$ è $\left(\mathrm{n}^{0} 31\right)$ $5184=\frac{12960 \cdot 2}{\tilde{5}}$

\section{$\S 12$. Esaedri.}

36. Dall' enneaedro di $1^{\text {a }}$ specie $\left(E_{1}\right)(\S 10)$ si hanno i due tipi di poliedri (cfr. n..$^{\circ} 33$ ):

$$
\begin{aligned}
& a_{1} a_{2} a_{3} a_{4} a_{5} a_{6}, \\
& a_{1} a_{2} a_{3} a_{4} a_{5} a_{7}:
\end{aligned}
$$

il primo dei quali è $(18,0,2)^{6}$ e l'altro $(17,0,3)^{6}$. Ogni triedro di $3^{a}$ specie di $\left(E_{1}\right)$ entra in 2 di quelli e 18 di questi; e però in $\left(E_{1}\right)$ esistono $12=\frac{12 \cdot 2}{2}$ dei primi e $72=\frac{12 \cdot 18}{3}$ dei secondi. Per conseguenza il numero degli esaedri $(18,0,2)^{6}$ è $480=12.40$ e quello degli esaedri $(17,0,3)^{6}$ è $2880=72 \cdot 40$. Dall' enneaedro di $2^{i}$ specie $\left(E_{2}\right)(\S 10)$ nascono i tre tipi

$$
\begin{aligned}
& b_{1} b_{2} b_{3} b_{4} b_{5} b_{6}, \\
& b_{1} b_{2} b_{3} b_{4} b_{5} b_{7}, \\
& b_{1} b_{2} b_{4} b_{5} b_{7} b_{8},
\end{aligned}
$$

che sono ordinatamente $(18,0,2)^{6},(13,6,1)^{6},(12,8,0)^{6}$. Che i yentaedri $(18,0,2)^{6}$ che qui si ottengono, sieno di quelli che esistono in un enneaedro di $1^{\prime}$ specie, segue subito dall'osservare che l'esaedro $b_{1} b_{2} b_{3} h_{4} b_{5} b_{6}$ esiste nell'enneaedro di $1^{\text {a }}$ specie dedotto da $\left(E_{2}\right)$ col sostituire al triedro (di $3^{\text {d }}$ specie) $b_{7} b_{8} b_{9}$ il suo conjugato (n. ${ }^{0} 13$ ). Il numero degli esaedri $(12,8,0)^{6}$ è $4320=$ $(3 \cdot 3 \cdot 3) \cdot 160$ : e quello degli esaedri $(13,6,1)^{6}$ è $8640=(2 \cdot 3 \cdot 9) \cdot 160$.

Dall' ettaedro principale $(P)(\S 10)$ si hanno i tipi

$$
\begin{aligned}
& z x_{1} x_{2} x_{3} y_{1} y_{2}, \\
& x_{1} x_{2} x_{3} y_{1} y_{2} y_{3}:
\end{aligned}
$$

il primo è $(10,10,0)^{6}$ e il secondo $(12,8,0)^{6}$. Il poliedro $(10,10,0)^{6}$, contiene un (solo) tetraedro $z x_{1} x_{2} x_{3}$, di cui i quattro triedri sono di $2^{\iota}$ specie (n. 26): ma da un tetraedro di tal sorta provengono 12 di quei poliedri, come 
si ricava dalle cose del n. ${ }^{\circ} 25\left(^{*}\right)$ : quindi il numero degli esaedri $(10,10,0)^{6}$ è (n. ${ }^{\circ}$ 24) $12960=1080 \cdot 12$. Questo essendo pure (n. $\left.{ }^{\circ} 33\right)$ il numero dei pentaedri $(6,4,0)^{5}$ ed esistendo in un esaedro $(10,10,0)^{6}$ due di tali pentaedri $\left(z x_{1} x_{2} y_{1} y_{2}, x_{1} x_{2} x_{3} y_{1} y_{2}\right)$, si ha che un pentaedro $(6,4,0)^{5}$ appartiene a due esaedri $(10,10,0)^{6}$.

Il pentaedro $(12,8,0)^{6}$ che proviene da un ettredro principale è di quelli che si trovano in un enneaedro di $2^{\mathrm{a}}$ specie, per la seguente proprietà.

37. Un esaedro di cui $i 20$ triedri sono 12 di $1^{a}$ specie e 8 di $2^{a}$ esiste in un enneaedro di $2^{a}$ specie.

In vero ciascun esaedro avente la detta proprietà contiene sei pentaedri $(6,4,0)^{5}$ : cioè $\left(\mathrm{n} .^{0} 36\right) b_{1} b_{2} b_{4} b_{5} b_{7} b_{8}$ i pentaedri $b_{1} b_{2} b_{4} b_{5} b_{7}, b_{1} b_{2} b_{4} b_{5} b_{8}, \ldots$ c $x_{1} x_{2} x_{3} y_{1} y_{2} y_{3}$ i pentaedri $x_{1} x_{2} x_{2} y_{1} y_{2}, x_{1} x_{2} x_{3} y_{1} y_{3}, \ldots$

Ora da un pentaedro $(6,4,0)$, ad es. dal pentaedro $a b c a^{\prime \prime} \alpha\left(\right.$ n. $\left.^{\circ} 34\right)$, ritornando nuovamente alle indicazioni de] $\S 9$ e ricordando le rette $(H)$, nascono sei (soli) esaedri

$$
\begin{array}{ll}
a b c a^{\prime \prime} \alpha b^{\prime \prime}, & a b c a^{\prime \prime} \alpha c^{\prime}, \\
a b c a^{\prime \prime} \alpha \beta, & a b c a^{\prime \prime} \alpha \gamma, \\
a b c a^{\prime \prime} \alpha \beta^{\prime \prime}, & a b c a^{\prime \prime} \alpha \gamma^{\prime \prime} .
\end{array}
$$

Di questi i primi quattro sono contenuti nell' enneaedro di $2^{\prime}$ specie $\left(E_{2}\right)(\S 9)$ e sono $\mathrm{i}$ due primi $(13,6,1)^{6}$ e $\mathrm{i}$ due secondi ciascuno con 12 triedri di $1^{2}$ specie e 8 di $2^{2}$. I due rimanenti $a b c a^{\prime \prime} \alpha \beta^{\prime \prime}, a b c a^{\prime \prime} \alpha \gamma^{\prime \prime}$ debbono essere $(10,10,0)^{\circ}$; il che risulta, o dall' osservare che non sono contenuti in alcun enneaedro (cfr. n..$\left.^{\circ} 35\right)$, o da una proprietà dell' esaedro $(10,10,0)^{6}$ avvertita alla fine del n. ${ }^{\circ} 36$. Adunque dei sei esaedri suddetti soltanto $a b c a^{\prime \prime} \alpha \beta$, $a b c a^{\prime \prime} \alpha . \gamma$ contengono 12 triedri di $1^{\text {a }}$ specie e 8 di $2^{a}$ ed esistono in $\left(E_{2}\right)$ $(\S 9)$ : c. d. d.

(*) Dal tetraedro $c a b d$ nascono i 12 esaedri

$$
\begin{aligned}
& \text { cabdmn, cabdmp, cabdnp, cabdmn, cabdmp', cabdn } p^{\prime} \text {, }
\end{aligned}
$$

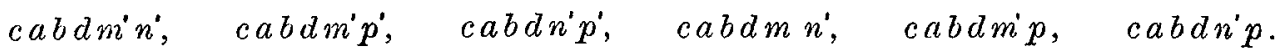

E qui si avverta che un esaedro $c a b d m n$ entra in 2 ettaedri

$$
\text { c.abd.nmp, dabc.nmp' }
$$

$e$ in ciascuno fignra collo stesso tipo. Invece il pentaedro $c a b m n$, che fa parte dell'esaedro c $a b d m n$, entra negli stessi due ettaedri, ma vi figura con tipo diverso. Ciò spiega l'apparente contraddizione dell'esscre, nello specchio del $\$ 14, j=1$ per il pentaedro $(6,4,0)^{5}$ nel tijo $z x_{1} x_{2} y_{1} y_{2}$ e $j=2$ per l'esaedro $z x_{1} x_{2} x_{3} y_{1} y_{2}$. 


\section{$\S$ 13. Ettaedri. Ottaedri.}

38. È facile vedere che un enneaedro di $1^{a}$ specie $\left(E_{1}\right)(\S 10)$ dà origine al solo tipo di ettaedri

$$
a_{1} a_{2} a_{3} a_{4} a_{5} a_{6} a_{7}
$$

(contenerite due triedri di $3^{\mathrm{a}}$ specie $a_{1} a_{2} a_{3}, a_{4} a_{5} a_{6}$ che non hanno alcun piano comune), e che in un enneaedro di $2^{\lambda}$ specie $\left(E_{z}\right)(\S 10)$ esistono $\mathrm{i}$ due tipi

$$
\begin{aligned}
& b_{1} b_{2} b_{3} b_{4} b_{5} b_{6} b_{7}, \\
& b_{1} b_{2} b_{3} b_{4} b_{5} b_{7} b_{8} .
\end{aligned}
$$

Questi tre ettaedri sono ordinatamente $(30,0,5)^{7},(24,9,2)^{7},(22,12,1)^{7}$ : ed

$$
\begin{array}{rlrlrl}
1440 & =(3 \cdot 12) \cdot 40 & \text { il numero degli ettaedri } & (30,0,5)^{7}, \\
1440 & =9 \cdot 160 & & & & \\
4320 & =(3 \cdot 3 \cdot 3) \cdot 160 & & & (24,9,2)^{7}, \\
& & &
\end{array}
$$

39. Infine dagli stessi due enneaedri menzionati nel numero precedente provengono ottaedri dei due tipi

$$
\begin{aligned}
& a_{1} a_{2} a_{3} a_{4} a_{5} a_{6} a_{7} a_{8} \\
& b_{1} b_{2} b_{3} b_{4} b_{5} b_{6} b_{7} b_{8}
\end{aligned}
$$

che sono $(48,0,8)^{8},(36,18,2)^{8}$. Il numero dei primi è $360=9.40$ e quelio dei secondi $1440=9 \cdot 160$.

\section{$\S 14$. Specchio rinssuntivo dei poliedri.}

40. Dai $\S \S 10,11,12,13$ (in particolare dai teoremi dei n.i $32,34,37$ ) discende la notevole proprietà: - Un poliedro è pienamente definito dai numeri $n, t_{1}, t_{2}, t_{3}\left(\mathrm{n} .^{\circ} 31\right)\left(^{*}\right)$ - Cioè: la specie di un poliedro è data dalla conoscenza dell'ordine, del numero dei triedri di $1^{a}$ specie, del numero di quelli di $2^{a}$ e del numero di quelli di $3^{a}$. In altre parole: $i$ poliedri, pei quali quei quattro numeri sono gli stessi, godono delle medesime proprietì.

41. Chiudiamo questo lavoro col riassumere nello specchio seguente i risultati precedentemente ottenuti sui poliedri. Tutti i possibili poliedri si distri-

(") Tre qualunque dei quali, per la relazione $t_{1}+t_{2}+t_{3}=\frac{n(n-1)(n-2)}{1.2 .3}$, determinano il quarto. 
buiscono in 27 specie [prescindendo dai sistemi di due piani (coppie o diedri), che formano una specie sola] e sono 3 di triedri, 4 di tetraedri, 7 di pentaedri, 5 di esaedri, 4 di ettaedri, 2 di ottaedri e 2 di ennenedri.

Nello specchio si trova anche l'indicazione dei tipi. Ma tale indicazione non ha valore in senso assoluto, $\mathrm{i}$ varî tipi di una stessa specie essendo poliedri identici (n. ${ }^{\circ} 40$ ). I tipi di un poliedro sono gli aspetti o i modi, ne' quali esso entra nei differenti poliedri principali $e$ in uno stesso poliedro principale. $\mathrm{E}$, per l'indicazione dei tipi, s'intendono rappresentati l'enneaedro di $1^{\text {a }}$ specie dalle notazioni $\left(E_{1}\right),(D)$, quello di $2^{2}$ specie da $\left(E_{2}\right)$, l'ettaedro principale da $(P)(\S 10)$ e il pentaedro principale dalla notazione

$$
c_{1} c_{2} c_{3} c_{4} c_{5} \text {. }
$$

Ad es., il pentaedro $(6,4,0)^{5}$ entra sotto l' aspetto o tipo $b_{1} b_{2} b_{4} b_{5} b_{7}$ nell'enneaedro di $2^{a}$ specie e nei due aspetti o tipi diversi $z x_{1} x_{2} y_{1} y_{2}, x_{1} x_{2} x_{3} y_{1} y_{2}$ nell' ettaedro principale.

Di fronte ad ogni tipo sono posti due numeri; uno $i$ dà il numero dei poliedri in quel tipo che esistono nel poliedro principale a cui il tipo stesso si riferisce, l'altro $j$ il numero di tali poliedri principali ai quali appartiene un poliedro in quel tipo.

Così, tenendo l'esempio precedente, cioè considerando pentaedri $(6,4,0)^{5}$, si ha che ne esistono 81 del tipo $b_{1} b_{2} b_{4} b_{5} b_{7}$ nell' enneaedro di $2^{2}$ specie e 1 di quel tipo entra in un solo tale enneaedro: che ne esistono 3 del tipo $z x_{1} x_{2} y_{1} y_{2}$ in un ettaedro principale e nuovamente 1 dello stesso tipo entra in un solo tale ettaedro: e infine che ne esistono 6 del tipo $x_{1} x_{2} x_{3} y_{1} y_{2}$ in un ettaedro principale e 1 fa parte di due tali ettaedri.

Il numero $i$ si trova facilmente. Il numero $j$ si calcola, osservando che, se $N$ è il numero dei poliedri di una specie, il numero $j \frac{N}{i}$ deve essere eguale a 40 (numero degli enneaedri di $1^{\text {a }}$ specie) se il tipo di quei poliedri, che si considera, appartiene all'enneaedro di $1^{2}$ specie; ovvero a 160 , o a 4320 , o a 216 , se il detto tipo appartiene invece all'enneaedro di $2^{a}$ specie o all' ettaedro principale 0 al pentaedro principale.

La somma dei numeri $i$ e la somma dei numeri $j$ di tutti i tipi di un poliedro di una data specie che entrano in un poliedro principale, sono rispettivamente il numero totale dei poliedri di quella specie che entrano nel poliedro principale considerato e il numero totale di tali poliedri principali ai quali appartiene un poliedro della stessa specie.

Ad es., $(640)^{5}$ entra $9(=3+6)$ volte in un ettaedro principale e appartiene a $3(=1+2)$ ettaedri principali.

Annali di Matematica, tomo XII. 


\begin{tabular}{|c|c|c|c|c|c|c|c|c|c|c|c|}
\hline $\begin{array}{c}\text { Po- } \\
\text { LIEDRI }\end{array}$ & Specie & $\begin{array}{l}\text { Nu- } \\
\text { MERo }\end{array}$ & $\begin{array}{c}\text { Tipo } \\
\text { (rispetto ai poliedri } \\
\text { principali) }\end{array}$ & $i$ & $j$ & $\begin{array}{c}\text { Po- } \\
\text { IIEDRT }\end{array}$ & Specie & $\begin{array}{l}\text { NU- } \\
\text { MERO }\end{array}$ & $\begin{array}{c}\text { TrPo } \\
\text { (rispetto ai poliedri } \\
\text { principali) }\end{array}$ & $i$ & $j$ \\
\hline 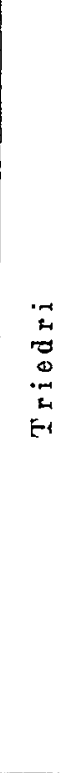 & $(1,0,0)^{3}$ & 2880 & $\begin{array}{l}\left\{\begin{array}{c}a_{1} a_{2} a_{4} \\
b_{1} b_{2} b_{4} \\
x_{1} x_{2} y_{1} \\
z x_{1} y_{2}\end{array}\right. \\
\left\{\begin{array}{l}b_{1} b_{4} b_{7} \\
x_{1} x_{2} x_{3} \\
z x_{1} x_{2} \\
z x_{1} y_{1} \\
x_{1} y_{2} y_{3}\end{array}\right.\end{array}$ & $\begin{array}{r}54 \\
12 \\
6 \\
27 \\
2\end{array}$ & $\begin{array}{r}1 \\
3 \\
18 \\
9 \\
2 \\
4 \\
12 \\
6\end{array}$ & 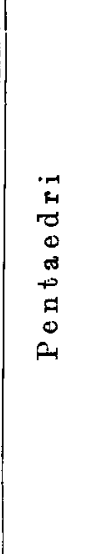 & $\begin{array}{c}(9,0,1)^{5} \\
(8,0,2)^{5} \\
(6,3,1)^{5} \\
(6,4,0)^{5} \\
(5,5,0)^{5} \\
(4,6,0)^{5} \\
(0,10,0)^{5}\end{array}$ & $\begin{array}{l}2880 \\
2160 \\
4320\end{array}$ & $\begin{array}{l}\left\{\begin{array}{l}a_{1} a_{2} a_{3} a_{4} a_{5} \\
b_{1} b_{2} b_{3} b_{4} b_{5} \\
a_{1} a_{2} a_{3} a_{4} a_{7} \\
b_{1} b_{2} b_{3} b_{4} b_{7}\end{array}\right. \\
\left\{\begin{array}{l}b_{1} b_{2} b_{4} b_{5} b_{7} \\
z x_{1} x_{2} y_{1} y_{2} \\
x_{1} x_{2} x_{3} y_{1} y_{2} \\
z x_{1} x_{2} y_{1} y_{3} \\
z x_{1} x_{2} x_{3} y_{1} \\
c_{1} c_{2} c_{3} c_{4} c_{5}\end{array}\right.\end{array}$ & $\begin{array}{r}72 \\
18 \\
54 \\
27 \\
81 \\
3 \\
6 \\
6 \\
6 \\
-\end{array}$ & $\begin{array}{r}2 \\
5 \\
4 \\
-\end{array}$ \\
\hline & $(0,0,1)^{3}$ & 2160 & $\left\{\begin{array}{l}a_{1} a_{2} a_{4} a_{5} \\
b_{1} b_{2} b_{4} b_{5} \\
x_{1} x_{2} y_{1} y_{2}\end{array}\right.$ & $\begin{array}{r}54 \\
27 \\
3\end{array}$ & $\begin{array}{l}1 \\
2 \\
2\end{array}$ & $\begin{array}{l}\cdot-1 \\
3 \\
0 \\
0 \\
0 \\
0 \\
0 \\
0\end{array}$ & $\begin{array}{l}(18,0,2)^{6} \\
(17,0,3)^{6} \\
(13,6,1)^{6} \\
(12,8,0)^{6} \\
(10,10,0)^{6}\end{array}$ & $\begin{array}{r}480 \\
2880 \\
8640 \\
4320 \\
12960\end{array}$ & $\begin{array}{l}\left\{\begin{array}{l}a_{1} a_{2} a_{3} a_{4} a_{5} a_{6} \\
b_{1} b_{2} b_{3} b_{4} b_{5} b_{6} \\
a_{1} a_{2} a_{3} a_{4} a_{5} a_{7} \\
b_{1} b_{2} b_{3} b_{4} b_{5} b_{7}\end{array}\right. \\
\left\{\begin{array}{l}b_{1} b_{2} b_{4} b_{5} b_{7} b_{8} \\
x_{1} x_{2} x_{3} y_{1} y_{2} y_{3}\end{array}\right. \\
z x_{1} x_{2} x_{3} y_{1} y_{2}\end{array}$ & $\begin{array}{r}12 \\
3 \\
72 \\
54 \\
27 \\
1 \\
6\end{array}$ & $\begin{array}{l}1 \\
1 \\
1 \\
1 \\
1 \\
2\end{array}$ \\
\hline \multirow[t]{3}{*}{ 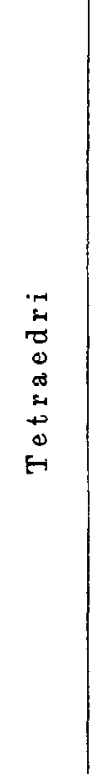 } & $(4,0,0)^{4}$ & 2880 & 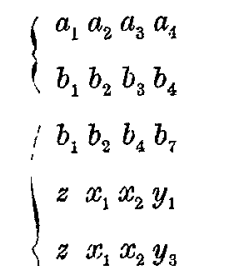 & $\begin{array}{r}72 \\
18 \\
81 \\
12 \\
6\end{array}$ & $\begin{array}{l}1 \\
1 \\
1 \\
4 \\
2\end{array}$ & 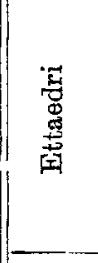 & $\left(\begin{array}{c}(90,0,5)^{7} \\
(24,9,2)^{7} \\
(22,12,1)^{7} \\
(18,17,0)^{7}\end{array}\right.$ & $\begin{array}{l}1440 \\
1440 \\
4320 \\
4320\end{array}$ & $\begin{array}{l}a_{1} a_{2} a_{3} a_{4} a_{5} a_{6} a_{7} \\
b_{1} b_{2} b_{3} b_{4} b_{5} b_{6} b_{7} \\
b_{1} b_{2} b_{3} b_{4} b_{5} b_{7} b_{8} \\
z x_{1} x_{2} x_{3} y_{1} y_{2} y_{3}\end{array}$ & $\begin{array}{r}36 \\
9 \\
27 \\
-\end{array}$ & $\begin{array}{r}1 \\
1 \\
1 \\
-\end{array}$ \\
\hline & & & $\begin{array}{l}x_{1} x_{2} x_{3} y_{1} \\
x_{1} x_{2} y_{1} y_{3}\end{array}$ & $\begin{array}{l}6 \\
6\end{array}$ & $\begin{array}{l}2 \\
2\end{array}$ & 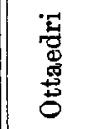 & $\begin{array}{c}(48,0,8)^{8} \\
(36,18,2)^{8}\end{array}$ & $\begin{array}{r}360 \\
1440\end{array}$ & $\begin{array}{l}a_{1} a_{2} a_{3} a_{4} a_{5} a_{6} a_{7} a_{8} \\
b_{1} b_{2} b_{3} b_{4} b_{5} b_{6} b_{7} b_{8}\end{array}$ & $\begin{array}{l}9 \\
9\end{array}$ & 1 \\
\hline & $(0,4,0)^{4}$ & 1080 & $\left\{\begin{array}{l}z x_{1} x_{2} x_{3} \\
c_{1} c_{2} c_{3} c_{3}\end{array}\right.$ & $\begin{array}{l}2 \\
5\end{array}$ & $\begin{array}{l}8 \\
1\end{array}$ & 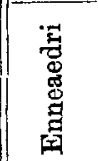 & $\begin{array}{l}(72,0,12)^{9} \\
(54,27,3)^{9}\end{array}$ & $\begin{array}{r}40 \\
160\end{array}$ & $\begin{array}{l}a_{1} a_{2} a_{3} a_{4} a_{5} a_{6} a_{7} a_{8} a_{9} \\
b_{1} b_{2} b_{3} b_{4} b_{5} b_{6} b_{7} b_{8} b_{9}\end{array}$ & - & - \\
\hline
\end{tabular}

Pavia, 1884.

Fine del Tomo XiI. ${ }^{\circ}$ (Serie II. ${ }^{a}$ ) 